\title{
Linee guida per le indagini geofisiche - I parte
}

Claudio Rossi

Presidente dell'Associazione Società di Geofisica - c/o CGT, San Giovanni Valdarno (AR)

rossi@sgg.it

\section{Presentazione}

A nome dell'Associazione delle Società di Geofisica (ASG) e mio personale, in primo luogo, voglio ringraziare la Redazione della rivista Acque Sotterranee-Italian Journal of Groundwater per averci ospitato sulle sue prestigiose pagine.

L'ASG è stata costituita nel gennaio del 2010, grazie all'idea di alcune imprese ed alcuni professionisti ai quali, dopo un breve periodo, se ne sono aggiunti molti altri. L'iniziativa è nata grazie all'impegno di Società che esistono (e resistono nonostante tutto) nel settore delle indagini geofisiche da moltissimo tempo (fin dal lontano 1974).

L'obiettivo dell'Associazione è quello di diffondere in modo capillare le metodologie geofisiche che possono, se ben eseguite, costituire un fondamentale supporto: allo studio del sottosuolo, dell'ambiente (nel suo complesso), alla progettazione e al controllo delle opere.

Negli ultimi anni abbiamo constatato il proliferare di soggetti che offrono prestazioni geofisiche non sempre adeguate alle problematiche da affrontare od utilizzando configurazioni lacunose; per questo motivo tutti insieme ci siamo impegnati a produrre le LINEE GUIDA PER LE INDAGINI GEOFISICHE per fornire uno strumento che possa essere utile sia agli esecutori che ai fruitori di tali prestazioni.

Queste verranno pubblicate in due parti.

La prima, presente su questo numero prende in esame "Prescrizioni di carattere generale" (progetto delle indagini, inizio lavori, direzione del cantiere, indagini in garanzia di qualità, documentazione delle indagini, ubicazione e quota) e "Prospezioni Sismiche" (Prospezione sismica a rifrazione ad onde di compressione (onde P), Indagine sismica a rifrazione ad onde di taglio (onde SH), Analisi della attenuazione anelastica e determinazione del fattore di qualità da onde di volume, Indagine sismica a riflessione ad onde di compressione, Indagine sismica a riflessione ad onde di taglio (S), Prospezione sismica in foro di sondaggio (Down-Hole), Prospezione sismica tra fori di sondaggio (Cross-Hole), Prospezione sismica con cono sismico, Prospezione geotomografica (fra sondaggi o fra sondaggio e superficie topografica), Prove per onde superficiali attive, prove per onde superficiali passive, Indagini di sismica passiva (HVSR).

La seconda sarà pubblicata nel numero 3/141 in uscita a Settembre 2015.

Nella convinzione di non aver prodotto un documento totalmente esaustivo, chiediamo a tutti $\mathrm{i}$ lettori di poterci far pervenire commenti e consigli al fine di poter rendere tali linee guida totalmente condivise ed utilizzate.

\author{
dr. Claudio Rossi \\ Presidente dell'Associazione Società di Geofisica
}




\title{
ASSOCIAZIONE \\ SOCIETA' DI GEOFISICA
}

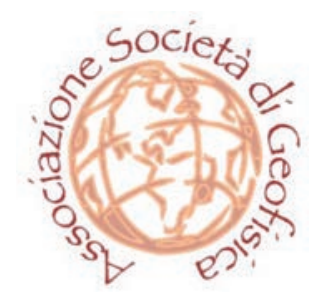

\section{LINEE GUIDA PER INDAGINI GEOFISICHE}

\author{
Associazione società di geofisica \\ c/o CGT - Via dei Vetri Vecchi, n ${ }^{\circ} 34$ \\ 52027 SAN GIOVANNI VALDARNO (AR)
}

Www.associazionegeofisica.org

info@associazionegeofisica.org 


\section{INDICE}

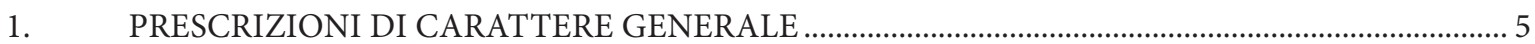

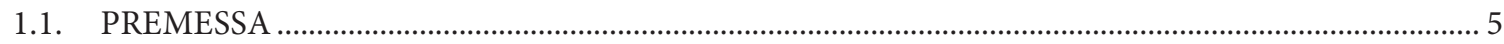

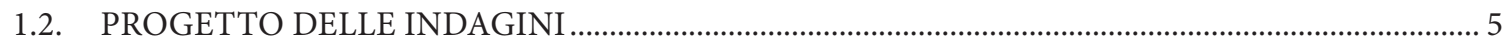

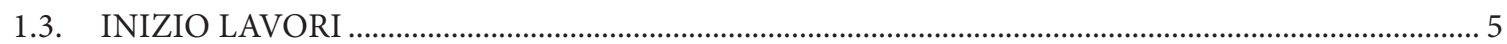

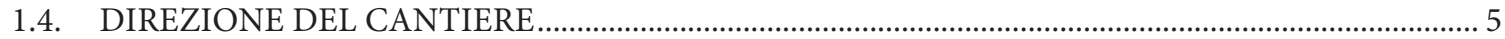

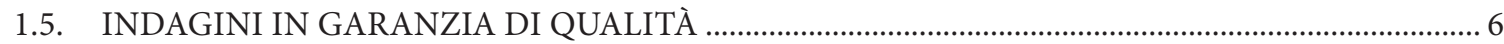

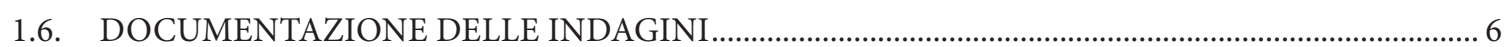

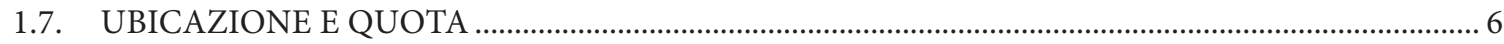

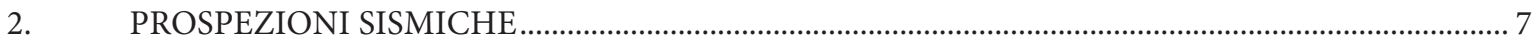

2.1 PROSPEZIONE SISMICA A RIFRAZIONE AD ONDE DI COMPRESSIONE (ONDE P) .................... 7

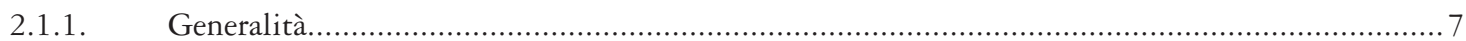

2.1.2. Normative e specifiche di riferimento ........................................................................ 7

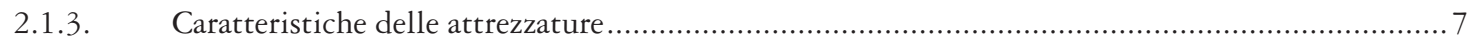

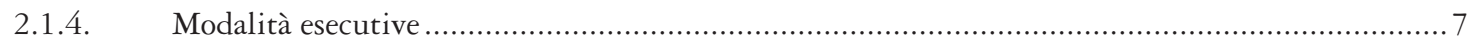

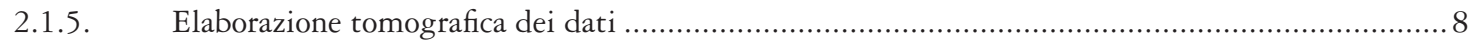

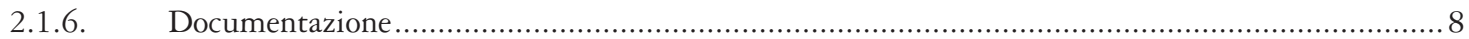

2.1.7. Rilievo topografico del piano completo di prospezione sismica........................................ 8

2.2. INDAGINE SISMICA A RIFRAZIONE AD ONDE DI TAGLIO (ONDE SH) .................................. 10

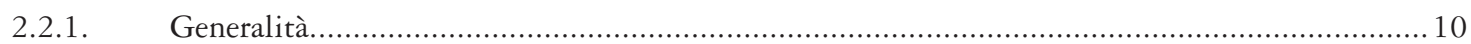

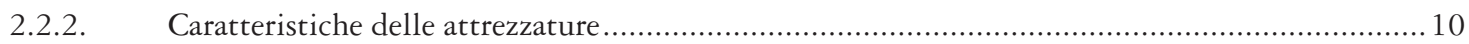

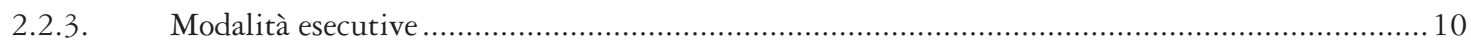

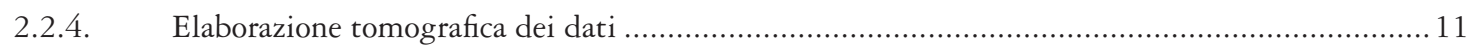

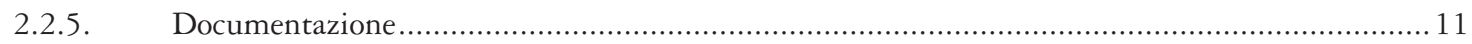

2.2.6. Rilievo topografico del piano completo di prospezione sismica ........................................ 11

2.3. ANALISI DELLA ATTENUAZIONE ANELASTICA E DETERMINAZIONE DEL

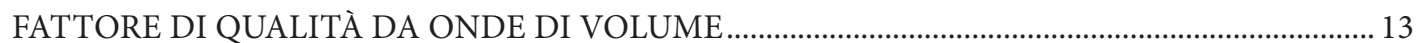

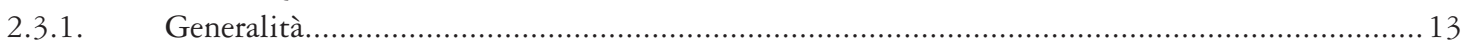

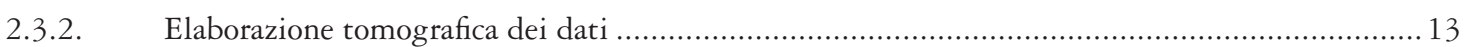

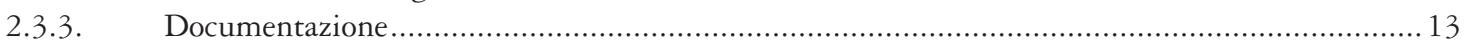

2.4. INDAGINE SISMICA A RIFLESSIONE AD ONDE DI COMPRESSIONE ....................................15

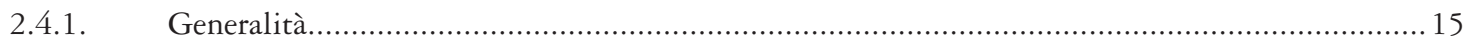

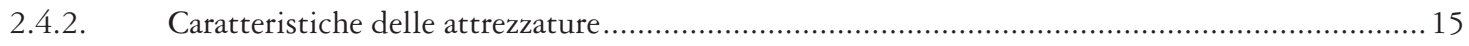

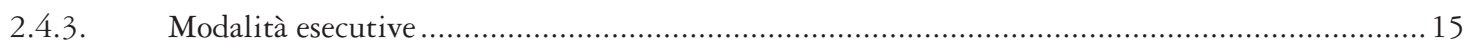

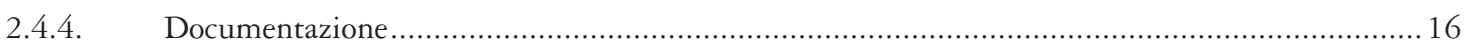

2.4.5. Rilievo topografico del piano completo di prospezione sismica........................................... 16

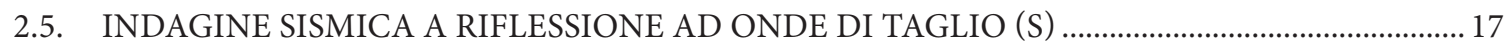

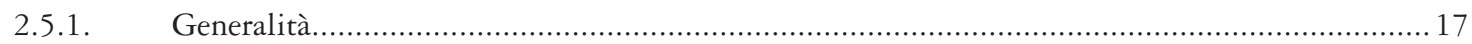

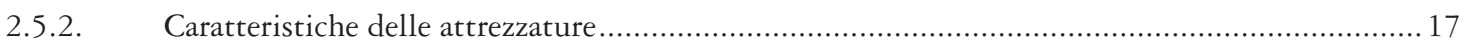

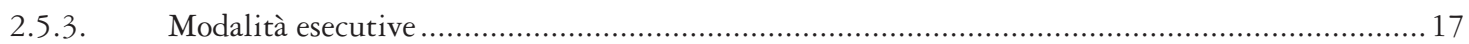

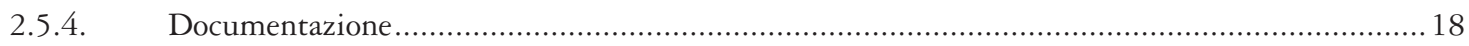

2.5.5. Rilievo topografico del piano completo di prospezione sismica ........................................ 18 
2.6. PROSPEZIONE SISMICA IN FORO DI SONDAGGIO (DOWN-HOLE) ……………....................... 19

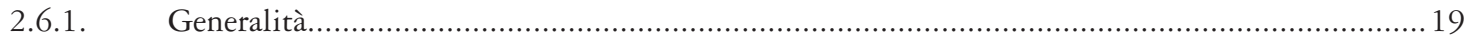

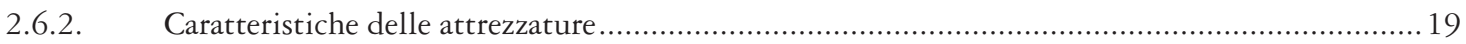

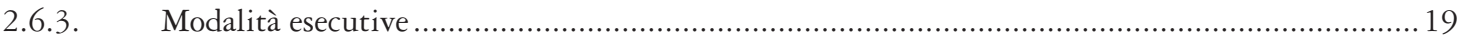

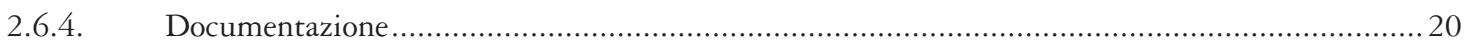

2.7. PROSPEZIONE SISMICA TRA FORI DI SONDAGGIO (CROSS-HOLE) …....................................... 21

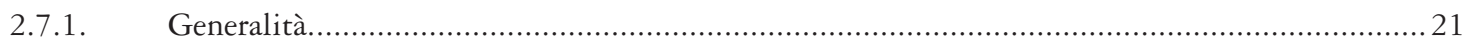

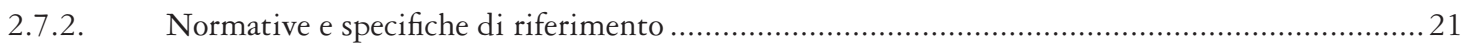

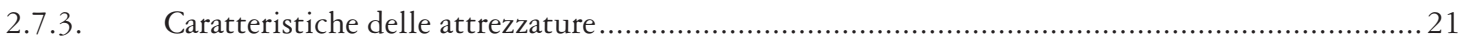

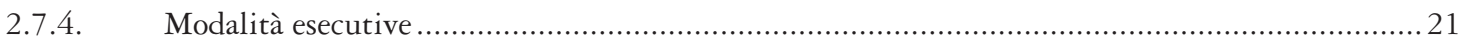

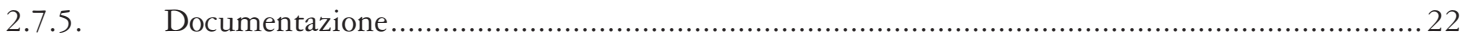

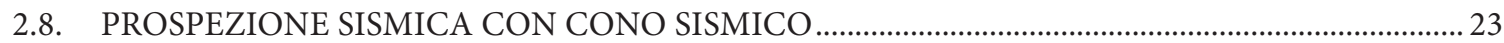

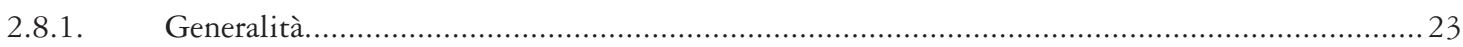

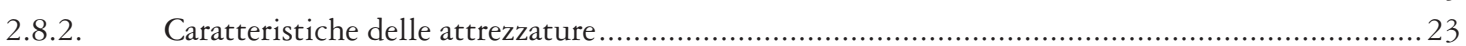

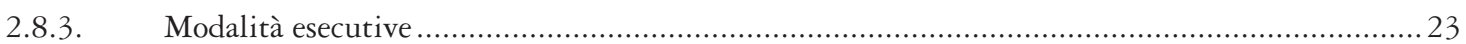

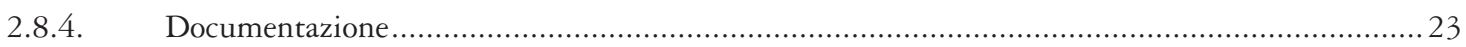

\subsection{PROSPEZIONE GEOTOMOGRAFICA (FRA SONDAGGI O FRA SONDAGGIO E}

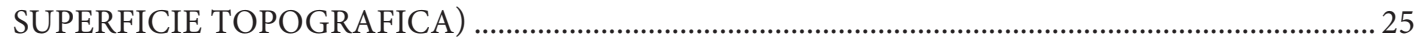

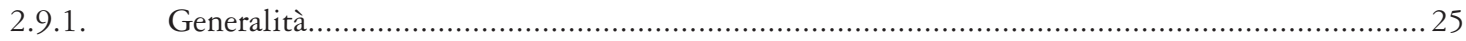

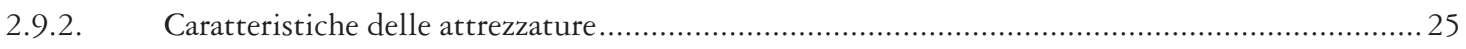

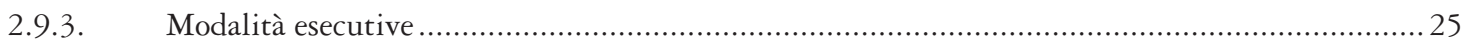

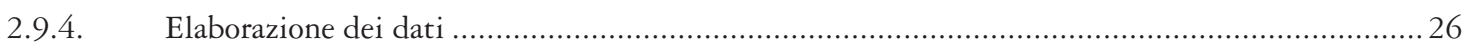

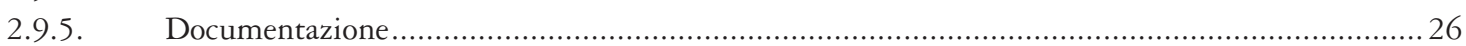

2.9.6. Rilievo topografico del piano completo di prospezione sismica.................................................26

2.10. PROVE PER ONDE SUPERFICIALI ATTIVE

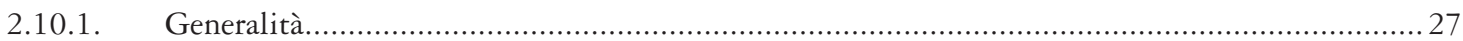

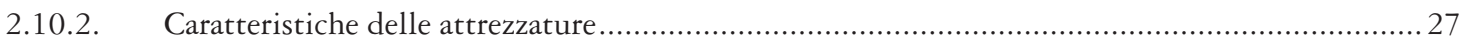

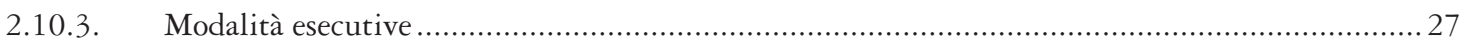

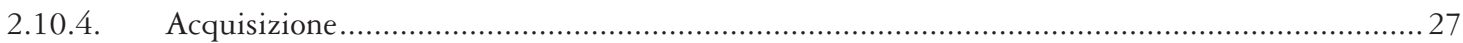

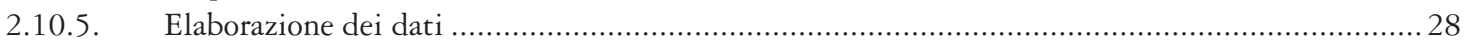

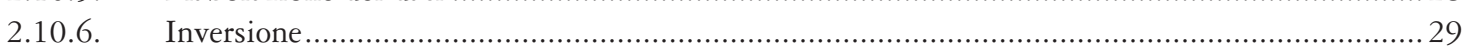

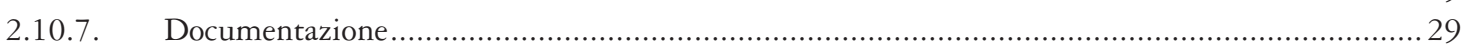

2.10.8. Rilievo topografico del piano completo di prospezione sismica................................................2

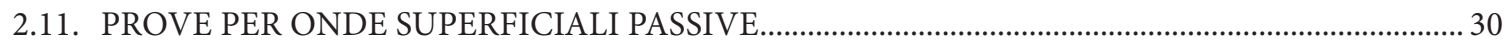

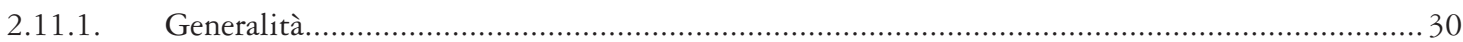

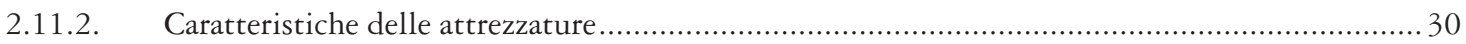

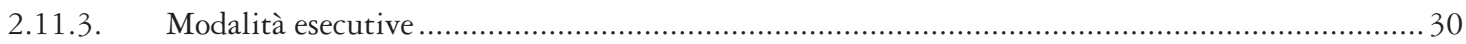

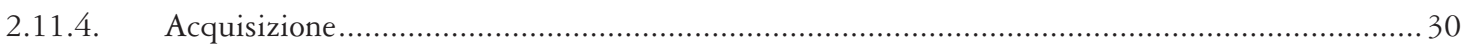

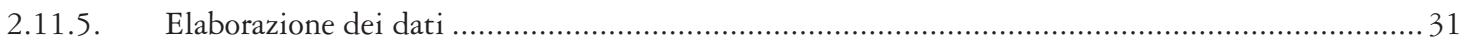

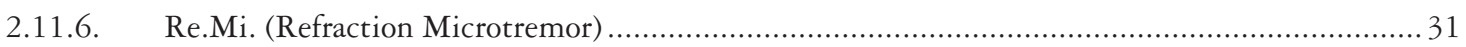

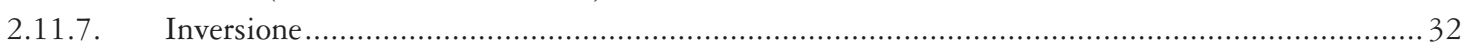

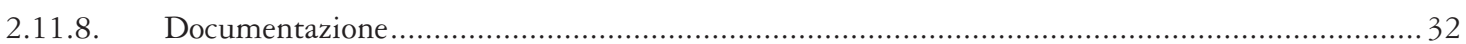

2.11.9. Rilievo topografico del piano completo di prospezione sismica................................................33

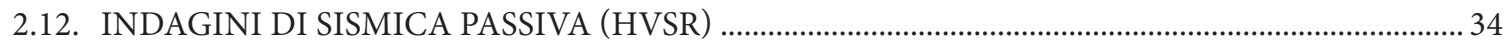

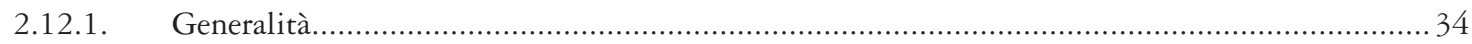

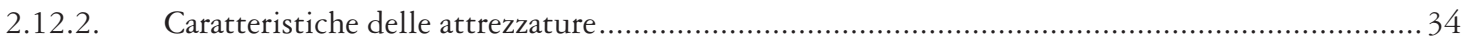

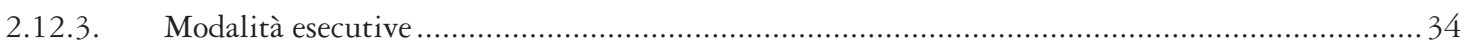

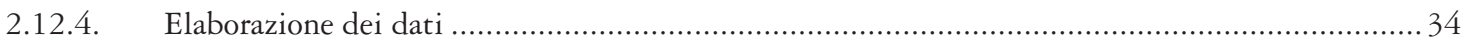

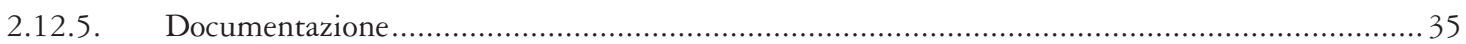




\section{PRESCRIZIONI DI CARATTERE GENERALE}

\subsection{Premessa}

Le presenti Specifiche Tecniche fanno parte integrante del contratto di appalto.

L'Impresa dovrà eseguire le attività in ottemperanza alle Leggi, ai regolamenti vigenti ed alle prescrizioni delle Autorità competenti, in conformità agli ordini che saranno impartiti dalla Direzione Lavori.

Le presenti Specifiche Tecniche determinano in modo prioritario le modalità di esecuzione e di valutazione dei lavori di indagine geofisica.

Nella esecuzione delle attività concernenti le indagini geofisiche si devono considerare inclusi oneri e costi per la documentazione fotografica delle attività svolte, per i carburanti e ogni altro materiale di consumo, i costi logistici e del personale, le attrezzature primarie, quelle accessorie e quanto necessario per l'esecuzione a perfetta regola d'arte di tutti i lavori oggetto del Contratto.

Sono esclusi specificatamente gli oneri e costi per il picchettamento e il rilievo plano-altimetrico e per la bonifica da residuati bellici; tali oneri, sostenuti dall'Impresa e qualora ad essa demandati, potranno essere compensati se documentati e concordati con la D. L..

L'Impresa esecutrice si impegna a visitare preventivamente i siti considerando le problematiche connesse con lo svolgimento a regola d'arte dei lavori d'indagine e ad evidenziare le proprie osservazioni al progetto delle indagini nella compilazione del Piano Qualità di Commessa.

\subsection{Progetto delle indagini}

Le presenti Specifiche Tecniche hanno carattere generale. Esse saranno integrate, per l'appalto, da un progetto delle indagini, fornito dalla Direzione Lavori, che indicherà il numero, la tipologia delle indagini geofisiche da eseguirsi.

I lavori di indagine geofisica dovranno essere realizzati dall'Impresa con personale, modalità operative ed attrezzature conformi alle prescrizioni delle presenti Specifiche Tecniche, senza apportare variazioni che non siano state preventivamente concordate ed approvate dalla Direzione Lavori.

\subsection{Inizio lavori}

La Direzione Lavori comunicherà all'Impresa la data d'inizio dei lavori in forma scritta, quando non preventivamente indicato nel progetto delle indagini. Prima dell'inizio dei lavori l'Impresa dovrà presentare, se richiesto dalla Direzione Lavori, il Piano Qualità di Commessa (PQC) relativo al lavoro affidatole, qualora l'impresa sia in Assicurazione di qualità.

In tutti gli altri casi dovrà essere presentato comunque il Piano Operativo di Commessa, indicando le attrezzature che dovranno essere utilizzate per lo sviluppo dei lavori, lo sviluppo temporale delle indagini, i nomi degli operatori ed il responsabile del cantiere e le esperienze acquisite.

\subsection{Direzione del Cantiere}

L'Impresa dovrà assicurare la presenza a tempo pieno in can- tiere di un geologo con almeno due anni di esperienza nel campo specifico delle indagini geofisiche, al quale verrà affidato il coordinamento dei lavori di indagine.

Il geologo sarà responsabile della corretta esecuzione delle attività, della realizzazione delle indagini e dei certificati di prova, della raccolta dei dati e della loro trasmissione alla Direzione Lavori nonché della fornitura di eventuali documenti integrativi che potrebbero servire alla definizione particolareggiata delle attrezzature e delle modalità esecutive.

Nel caso in cui le indagini vengano svolte in Garanzia di Qualità, l' Impresa indicherà un geologo con funzione di Responsabile Controllo Qualità (RCQ) presente in cantiere a tempo pieno.

Al geologo di cantiere (RCQ) sarà affidato il coordinamento dei lavori di indagine e sarà responsabile della corretta esecuzione delle varie fasi dell' indagine. In particolare egli dovrà adottare quanto necessario per prevenire il verificarsi di non conformità, gestire le eventuali non conformità in collaborazione con il Responsabile del Servizio Garanzia di Qualità (RSGQ) e/o con la Direzione Lavori, proporre quanto ritenuto più idoneo per la risoluzione delle non conformità, raccogliere i dati da fornire alla Direzione Lavori, organizzare le varie attività in sito, mantenere i rapporti con la Direzione Lavori per tutto quanto riguarda il corretto svolgimento delle indagini.

\subsection{Indagini in garanzia di qualità}

La Direzione Lavori si riserva il diritto di individuare una Impresa certificata secondo la normativa UNI EN ISO 9001: 2008 qualora lo ritenga opportuno, a seguito delle problematiche legate alla progettazione dell'opera.

La certificazione dovrà essere emessa da un Organismo internazionale esterno ed autonomo, riconosciuto in Italia dal SINCERT.

L'emissione della Certificazione di qualità implica che le procedure aziendali, i processi di produzione, la documentazione ed ogni altro aspetto della gestione della qualità adottate dall' Impresa stessa siano tali da garantire alla Società una superiore qualità del servizio offerto.

\subsection{Documentazione delle Indagini}

La documentazione preliminare del lavoro svolto verrà progressivamente aggiornata nel corso dei lavori e sarà resa disponibile e trasmessa alla Direzione Lavori quando richiesta e comunque a fine lavoro.

La documentazione in forma definitiva sarà presentata non oltre 20 giorni solari dal completamento dei lavori, salvo diversa prescrizione.

Tutta la documentazione finale dovrà essere fornita in formato digitale, secondo gli standards concordati con la Direzione Lavori.

\subsection{Ubicazione e Quota}

Lubicazione e la quota di ciascun punto di indagine dovrà essere determinata mediante rilevazione delle coordinate $\mathrm{x}, \mathrm{y}$, z eseguite tramite rilevazione con sistema GPS e se espressamente richiesto, l'ubicazione di ciascun punto di indagine 
dovrà risultare da un apposito rilievo topografico eseguito dall'Impresa.

In tal caso la posizione planimetrica di ciascun punto di indagine sarà definita rispetto a capisaldi forniti dalla Direzione Lavori e la quota di ciascun punto di indagine sarà definita rispetto al livello medio marino o al riferimento locale fornito dalla Società.

\section{PROSPEZIONI SISMICHE 2.1. Prospezione sismica a rifrazione ad onde di} 2.1.1. Generalità

L'indagine sismica a rifrazione consiste nella registrazione dei tempi di arrivo delle onde di compressione $(\mathrm{P})$, create allo scopo tramite opportuna energizzazione, e rifratte dalle superfici che costituiscono contrasti di impedenza del sottosuolo. La registrazione si realizza attraverso uno stendimento di geofoni disposti a intervalli regolari lungo il profilo da indagare. L'equidistanza tra i geofoni ed il loro numero dipendono dal dettaglio e dal target (profondità di indagine richiesta).

La misura dei tempi di arrivo delle onde $\mathrm{P}$ ai diversi geofoni permette di ricostruire l'andamento e la profondità degli orizzonti rifrattori presenti nel sottosuolo e, nel caso di misura anche delle onde secondarie o di taglio (S), di calcolare le caratteristiche elastiche dinamiche dei terreni e degli ammassi rocciosi investigati.

\subsubsection{Normative e specifiche di riferimento}

ASTM D 5777 - 95 - Standard Guide for Using the Seismic Refraction Method for Subsurface Investigation.

\subsubsection{Caratteristiche delle attrezzature}

L'attrezzatura di prova consigliata dovrà essere costituita dai seguenti componenti:

- $\quad$ sismografo minimo a 24 canali, con possibilità di stack degli impulsi sismici, filtri analogici e digitali programmabili (filtri attivi tipo bigh pass, band pass e band reject), guadagno verticale del segnale (in ampiezza) e sensibilità tra 6 e 92 decibel, registrazione dei dati in digitale per elaborazioni successive con formato in uscita minimo a 16 bit;

- minimo 24 geofoni verticali a frequenza propria variabile tra 8 e $30 \mathrm{~Hz}$;

- $\quad$ sistema di energizzazione adeguato alla lunghezza dei tiri da realizzare; potrà essere costituito da:

- martello strumentato lasciato cadere con energia su una piastra metallica appoggiata al suolo;

- cannoncino sismico;

- energizzatori oleopneumatici e/o gravimetrici trainati e/o trasportati;

- cariche di esplosivo.

È necessario che il tipo di energizzatore utilizzato permetta dopo qualche stack (massimo 5) di determinare inconfutabilmente i primi arrivi su tutti i ricevitori dell'allineamento

\subsubsection{Modalità esecutive}

La "copertura" dei tiri sullo stendimento dovrà essere tale da consentire una corretta e dettagliata ricostruzione del campo di velocità locale fino alle profondità stabilite dal progetto delle indagini o dalla Direzione Lavori ed in ogni caso dovranno essere ogni 3, 4 stazioni riceventi; nel caso in cui non sia prevista una elaborazione tomografica i tiri dovranno essere anche esterni allo stendimento di almeno 2 posizioni per ogni estremo.

Generalmente per ogni stendimento la profondità massima raggiunta dalla prospezione sismica è funzione sia delle velocità sismiche dei singoli strati sia della copertura dei tiri sismici.

Indicativamente la profondità massima indagata è circa $1 / 4$ - 1/5 della lunghezza dei tiri sismici e per ottenere dei risultati ottimali occorre mantenere il target della ricerca entro i $2 / 3$ della profondità massima indagata.

Tale indicazione è adeguata sia per l'elaborazione sismica di tipo ordinario che tomografica.

La spaziatura delle stazioni geofoniche è funzione del dettaglio che si vuole ottenere in particolare nella sismica tomografica: indicativamente la spaziatura intergeofonica deve variare tra $1 / 4$ ed $1 / 5$ della profondità del target.

Ad esempio se la profondità del target è posta a

- $10 \mathrm{~m}$ la spaziatura dei geofoni ottimale sarà 2 - 2.5 metri $[10 / 4(5)=2.5(2.0) \mathrm{m}\}$;

- $20 \mathrm{~m}$ la spaziatura dei geofoni ottimale sarà $4-5$ metri $[20 / 4(5)=5(4) \mathrm{m}\}$

- $\quad 50 \mathrm{~m}$ la spaziatura dei geofoni ottimale sarà 10 - 12 metri $[50 / 4(5)=12(10) \mathrm{m}$;

- $100 \mathrm{~m}$ la spaziatura dei geofoni ottimale sarà di 20 - 25 metri $[100 / 4(5)=25(20) \mathrm{m}]$.

In entrambi i casi (sismica tomografica o ordinaria) si consiglia di non superare mai la spaziatura di $20 \mathrm{~m}$ perché si inficerebbe l'analisi della porzione superficiale (copertura allentata) la quale può determinare importanti variazioni sia dei parametri elastici del sottosuolo sia degli orizzonti se la sua determinazione è errata.

L'elaborazione dei dati dovrà essere realizzata mediante software ad elevata valenza diagnostica in grado di fornire $\mathrm{i}$ valori dei parametri di velocità, relativi ai rifrattori individuati, per ogni stazione geofonica (ad esempio software che utilizzi il metodo "GRM" - Generalized Reciprocal Method, Palmer '80)

\subsubsection{Elaborazione tomografica dei dati}

Se richiesto dal progetto delle indagini, l'elaborazione dovrà essere sviluppata tramite un'analisi con modellazione del sottosuolo su base anisotropica, la quale dovrà fornire, previa elaborazione con metodologie iterative R.T.C. (Ray Tracing Curvilineo) e algoritmi di ricostruzione tomografica (ad esempio con l'impiego di algoritmi ART - Algebric Reconstruction Technique, SIRT - Simultaneous Iterative Reconstruction Technique o ILST - Iterative Least Square Technique), il campo delle velocità del sottosuolo ad elevata densità di informazioni: le celle 
unitarie, di forma rettangolare, potranno avere dimensioni orizzontali (asse $\mathrm{x}$ ) e verticali (asse $\mathrm{z}$ ) pari a, rispettivamente, $1 / 3 \div 1 / 5$ e $1 / 5 \div 1 / 10$ della spaziatura tra i geofoni.

\subsubsection{Documentazione}

La documentazione di ciascuna indagine dovrà comprendere:

- informazioni generali (commessa, cantiere, ubicazione, data, nominativo dell'operatore);

- sismogrammi originali sia su supporto cartaceo e/o digitale;

- $\quad$ profili sismostratigrafici in scala adeguata con indicati i valori delle velocità delle onde di compressione $\left(\mathrm{V}_{\mathrm{P}}\right)$ calcolati per intervalli omogenei;

- elaborazione a isolinee o a campiture di colore delle velocità delle onde di compressione $\left(\mathrm{V}_{\mathrm{P}}\right)$ in caso di elaborazione tomografica;

- relazione conclusiva, elaborata in base ai risultati delle indagini svolte in cui saranno indicate le strumentazioni utilizzate, le metodologie operative, gli algoritmi di calcolo impiegati, tabelle e tavole ad integrazione e chiarimento delle analisi, procedure applicate, le risultanze finali ed interpretative;

- documentazione fotografica.

\subsubsection{Rilievo topografico del piano completo di prospezione sismica}

Ad integrazione dell'indagine sismica dovrà essere eseguito un rilievo topografico comprendente la determinazione planoaltimetrica delle ubicazioni dei geofoni delle basi sismiche a rifrazione, riferita a punti notevoli o ad elementi cartografici noti dell'area interessata. Le coordinate del rilievo topografico, dovranno essere inquadrate in un sistema di coordinate generale di progetto fornito dal Committente. Qualora quest'ultimo non risulti disponibile il suddetto rilievo sarà fornito in coordinate relative.

Tale rilievo dovrà essere corredato dai libretti di campagna dei rilievi ed informatizzazione dei dati nei formati digitali stabiliti dalla Società o dalla Direzione Lavori.

\subsection{Indagine sismica a rifrazione ad onde di taglio (onde SH)}

\subsubsection{Generalità}

L'indagine sismica a rifrazione consiste nella registrazione dei tempi di arrivo delle onde di taglio $(\mathrm{SH})$, create allo scopo tramite opportuna energizzazione, e rifratte dalle superfici di discontinuità fisica del sottosuolo. La registrazione si realizza attraverso uno stendimento di geofoni orizzontali disposti a intervalli regolari lungo il profilo da indagare. L'equidistanza tra i geofoni e il loro numero dipendono dal dettaglio e dal target (profondità di indagine richiesta).

La misura dei tempi di arrivo delle onde SH ai diversi geofoni permette di ricostruire l'andamento e la profondità degli orizzonti rifrattori presenti nel sottosuolo e, nel caso di misura anche delle onde di compressione (P), di calcolare le caratteristiche elastiche dinamiche dei terreni e degli ammassi rocciosi investigati.

\subsubsection{Caratteristiche delle attrezzature}

Lattrezzatura di prova dovrà essere costituita da almeno:

- sismografo a 24 canali, con possibilità di stack degli impulsi sismici, filtri analogici e digitali programmabili (filtri attivi tipo bigh pass, band pass e band reject), guadagno verticale del segnale (in ampiezza) e sensibilità tra 6 e 92 decibel, registrazione dei dati in digitale per elaborazioni successive con formato in uscita minimo a $16 \mathrm{bit}$; - 24 geofoni orizzontali a frequenza propria variabile tra 8 e $14 \mathrm{~Hz}$;

- $\quad$ sistema di energizzazione adeguato alla lunghezza dei tiri da realizzare; potrà essere costituito $\mathrm{da}$ :

- martello strumentato agente lateralmente su un blocco adeguatamente ancorato (per attrito radente) al terreno;

- energizzatori oleopneumatici e/o gravimetrici trainati e/o trasportati (pendoli) che producono onde di taglio polarizzate sul piano orizzontale;

- cariche di esplosivo (solo in casi eccezionali).

È necessario che il tipo di energizzatore utilizzato permetta dopo qualche stack (massimo 5) di determinare inconfutabilmente i primi arrivi su tutti i ricevitori dell'allineamento.

\subsubsection{Modalità esecutive}

La "copertura" dei tiri sullo stendimento dovrà essere tale da consentire una corretta e dettagliata ricostruzione del campo di velocità locale fino alle profondità stabilite dal progetto delle indagini o dalla Direzione Lavori ed in ogni caso dovranno essere ogni 3, 4 stazioni riceventi; nel caso in cui non sia prevista una elaborazione tomografica i tiri dovranno essere anche esterni allo stendimento di almeno 2 posizioni per ogni estremo.

Per la corretta determinazione delle onde di taglio, sarà necessario realizzare per ogni punto di energizzazione anche la registrazione coniugata (rovesciata di $180^{\circ}$ sul piano orizzontale rispetto alla direzione individuata dallo stendimento) in tal modo sarà possibile determinare correttamente l'arrivo dell'onda di taglio (dove si avrà inversione di fase) sul sismogramma.

Generalmente per ogni stendimento la profondità massima raggiunta dalla prospezione sismica è funzione sia delle velocità sismiche dei singoli strati sia della lunghezza dei tiri sismici.

Indicativamente la profondità massima indagata è circa $1 / 4$ - 1/5 della lunghezza dei tiri sismici e per ottenere dei risultati ottimali occorre mantenere il target della ricerca entro i 2/3 della profondità massima indagata.

Tale indicazione è adeguata sia per l'elaborazione sismica di tipo ordinario che tomografica.

La spaziatura delle stazioni geofoniche è funzione del dettaglio che si vuole ottenere in particolare nella sismica tomografica: indicativamente la spaziatura intergeofonica può variare tra $1 / 4$ ed $1 / 5$ della profondità del target. 
Ad esempio se la profondità del target è posta a:

- $10 \mathrm{~m}$ la spaziatura dei geofoni ottimale sarà 2 - 2.5 metri $[10 / 4(5)=2.5(2.0) \mathrm{m}\}$

- 20 m la spaziatura dei geofoni ottimale sarà 4 - 5 metri $[20 / 4(5)=5(4) \mathrm{m}]$

- $\quad 50 \mathrm{~m}$ la spaziatura dei geofoni ottimale sarà 10 - 12 metri $[50 / 4(5)=12(10) \mathrm{m}]$

- 100 m la spaziatura dei geofoni ottimale sarà di 20 - 25 metri $[100 / 4(5)=25(20) \mathrm{m}]$.

In entrambi i casi (sismica tomografica o ordinaria) si consiglia di non superare mai la spaziatura di $20 \mathrm{~m}$ in quanto si inficerebbe l'analisi della porzione superficiale (copertura allentata) la quale può determinare importanti variazioni sia dei parametri elastici del sottosuolo sia degli orizzonti se la sua determinazione è errata.

L'elaborazione dei dati dovrà essere realizzata mediante software ad elevata valenza diagnostica in grado di fornire i valori dei parametri di velocità, relativi ai rifrattori individuati, per ogni stazione geofonica (ad esempio software che utilizzi il metodo "GRM" - Generalized Reciprocal Method, Palmer '80).

\subsubsection{Elaborazione tomografica dei dati}

Se richiesto dal progetto delle indagini, l'elaborazione dovrà essere sviluppata tramite un'analisi con modellazione del sottosuolo su base anisotropica, la quale dovrà fornire, previa elaborazione con metodologie iterative R.T.C. (Ray Tracing Curvilineo) e algoritmi di ricostruzione tomografica (ad esempio con l'impiego di algoritmi ART - Algebric Reconstruction Technique, SIRT - Simultaneous Iterative Reconstruction Technique o ILST - Iterative Least Square Technique), il campo delle velocità del sottosuolo ad elevata densità di informazioni: le celle unitarie, di forma rettangolare, potranno avere dimensioni orizzontali (asse x) e verticali (asse z) pari a, rispettivamente, $1 / 3 \div 1 / 5$ e $1 / 5 \div 1 / 10$ della spaziatura tra i geofoni

\subsubsection{Documentazione}

La documentazione di ciascuna indagine dovrà comprendere:

- informazioni generali (commessa, cantiere, ubicazione, data, nominativo dell'operatore);

- $\quad$ sismogrammi originali su supporto cartaceo e/o digitale;

- profili sismostratigrafici in scala adeguata con indicati i valori di velocità delle onde di taglio $\left(\mathrm{V}_{\mathrm{S}}\right)$ calcolati per intervalli omogenei;

- elaborazione a isolinee o a campiture di colore delle velocità delle onde di taglio $\left(\mathrm{V}_{\mathrm{S}}\right)$ in caso di elaborazione tomografica;

- relazione conclusiva, elaborata in base ai risultati delle indagini svolte in cui saranno indicate le strumentazioni utilizzate, le metodologie operative, gli algoritmi di calcolo impiegati, tabelle e tavole ad integrazione e chiarimento delle analisi, procedure applicate, le risultanze finali ed interpretative;

- documentazione fotografica.

\subsubsection{Rilievo topografico del piano completo di prospezione sismica}

Ad integrazione dell'indagine sismica dovrà essere eseguito un rilievo topografico comprendente la determinazione planoaltimetrica delle ubicazioni dei geofoni delle basi sismiche a rifrazione, riferita a punti notevoli o ad elementi cartografici noti dell'area interessata. Le coordinate del rilievo topografico, dovranno essere inquadrate in un sistema di coordinate generale di progetto fornito dal Committente. Qualora quest'ultimo non fosse disponibile il suddetto rilievo sarà fornito in coordinate relative.

Tale rilievo dovrà essere corredato dai libretti di campagna dei rilievi ed informatizzazione dei dati nei formati digitali stabiliti dalla Società o dalla Direzione Lavori.

\subsection{Analisi della attenuazione anelastica $e$ determinazione del fattore di qualità da onde di volume}

\subsubsection{Generalità}

Consiste in una elaborazione dei dati ottenuti da prospezioni sismiche a rifrazione ad onde di compressione (onde P) e/o onde di taglio (onde $\mathrm{S}$ ) in grado di determinare i parametri di Attenuazione Anelastica e Fattore Qualità dei terreni o degli ammassi rocciosi investigati.

Il fattore di qualità $Q$ è correlato al coefficiente di attenuazione attraverso la seguente relazione:

$$
l / Q=a \times V_{p} / p \times f
$$

con

$a=$ coefficiente di attenuazione;

$V_{p}=$ velocità delle onde di compressione;

$f=$ frequenza dominante dell'analisi sismica.

Il coefficiente di attenuazione esprime l'entità del fenomeno di assorbimento dell'energia sismica in relazione allo specifico divario rispetto alla condizione di perfetta elasticità nei diversi materiali in cui l'onda sismica si propaga.

A seguito dell'attenuazione le componenti ad alta frequenza degli impulsi sismici si estinguono più rapidamente di quelli a bassa frequenza; la misura di $1 / \mathrm{Q}$, proporzionale alla frazione di perdita dell'energia per ciclo d'onda sinusoidale, contribuisce a fornire informazioni sulle caratteristiche del mezzo attraversato (frequenza di fratturazione, grado di saturazione, ecc.).

I valori numerici prodotti dall'elaborazione tomografica in velocità, attenuazione e Fattore Qualità saranno trasformati in informazioni grafiche e cromatiche bidimensionali e tridimensionali tramite software di trasferimento dati su un sistema C.A.D.

L'elaborazione dei dati dovrà essere realizzata mediante software ad elevata valenza diagnostica in grado di fornire $\mathrm{i}$ valori dei parametri di Attenuazione e Fattore Qualità, relativi ai rifrattori individuati e per ogni stazione geofonica utilizzando l'algoritmo del "Rapporto Spettrale". 


\subsubsection{Elaborazione tomografica dei dati}

Se richiesto dal progetto delle indagini, l'elaborazione dovrà essere sviluppata tramite un'analisi con modellazione del sottosuolo su base anisotropica, la quale dovrà fornire, previa elaborazione con metodologie iterative R.T.C. (Ray Tracing Curvilineo) e algoritmi di ricostruzione tomografica (ad esempio con l'impiego di algoritmi ART - Algebric Reconstruction Technique, SIRT - Simultaneous Iterative Reconstruction Technique o ILST - Iterative Least Square Technique), il campo delle velocità del sottosuolo ad elevata densità di informazioni: le celle unitarie, di forma rettangolare, potranno avere dimensioni orizzontali (asse $\mathrm{x}$ ) e verticali (asse $\mathrm{z}$ ) pari a, rispettivamente, $1 / 3 \div 1 / 5$ e $1 / 5 \div 1 / 10$ della spaziatura tra i geofoni

\subsubsection{Documentazione}

La documentazione di ciascuna indagine dovrà comprendere:

- informazioni generali (commessa, cantiere, ubicazione, data, nominativo dell'operatore);

- profili sismostratigrafici in scala adeguata con indicati i parametri dei Fattori Qualità calcolati per intervalli omogenei;

- elaborazione a isolinee o a campiture di colore in caso di elaborazione tomografica;

- relazione conclusiva, elaborata in base ai risultati delle indagini svolte in cui saranno indicati:

- gli algoritmi di calcolo impiegati, tabelle e tavole ad integrazione e chiarimento delle analisi;

- la caratterizzazione su base geofisica ottenuta da correlazione delle risultanze delle analisi ad onde di compressione (VP) e o taglio (VS) e Attenuazione onde di compressione (aP) e di taglio (aS) e Fattore Qualità delle onde di compressione (QP) e delle onde di taglio (QS);

- le risultanze finali ed interpretative.

\subsection{Indagine sismica a riflessione ad onde di compressione}

\subsubsection{Generalità}

L'indagine sismica a riflessione consiste nell' energizzazione del sottosuolo e nella registrazione degli arrivi delle onde di compressione (onde P) riflesse, in corrispondenza di geofoni verticali disposti secondo un allineamento con interassi tra $i$ geofoni e lunghezza totale dello stendimento tali da permettere una adeguata profondità di indagine.

La misura dei tempi di arrivo delle onde $\mathrm{P}$ ai diversi geofoni permette di ricostruire l'andamento e la profondità delle diverse discontinuità sismiche che costituiscono delle superfici riflettenti.

\subsubsection{Caratteristiche delle attrezzature}

Lattrezzatura di prova consigliata dovrà essere costituita dai seguenti componenti:

- $\quad$ sismografo minimo a 24 canali, con possibilità di stack degli impulsi sismici, filtri analogici e digitali program- mabili (filtri attivi tipo high pass, band pass e band reject), guadagno verticale del segnale (in ampiezza) e sensibilità tra 6 e 92 decibel, registrazione dei dati in digitale per elaborazioni successive con formato in uscita minimo a 16 bit;

- minimo 24 geofoni verticali a frequenza propria variabile tra 25 e $100 \mathrm{~Hz}$;

- Sistema di energizzazione adeguato alla profondità di indagine che potrà essere costituito da:

- martello strumentato lasciato cadere con violenza su una piastra metallica appoggiata al suolo;

- cannoncino sismico;

- energizzatori oleopneumatici e/o gravimetrici trainati e/o trasportati;

- cariche di esplosivo;

- vibroseis.

\subsubsection{Modalità esecutive}

Il rilievo sismico a riflessione dovrà essere eseguito per mezzo di stese lineari con geofoni posti ad intervalli regolari scelti in relazione alla profondità dell'obiettivo da raggiungere. In generale l'interdistanza tra i geofoni dovrà essere pari a $0.5,1$, 2.5, 5, 10 o 20 metri.

I punti di origine dell'energia dovranno essere ubicati o nel centro di simmetria del gruppo di registrazione (metodologia "Split Spread") oppure ad un estremo dello stendimento (metodologia "End On") o infine in posizione distanziata dai due punti precedenti fino ad un massimo di 30-50 metri dalla stesa ed in direzione parallela alla stesa stessa.

L'indagine e l'elaborazione dei dati dovranno garantire una "copertura multipla" minima del $1200 \%$.

La prospezione sismica a riflessione dovrà essere realizzata secondo criteri di "stacking orizzontale" mediante copertura multipla di stendimenti in ragione variabile da un minimo del $1200 \%$ secondo gli indirizzi e la finalità dell'indagine.

Per ottenere una copertura multipla del $1200 \%$ con un sismografo da 24 canali è necessario energizzare lungo linea con una interdistanza fra una energizzazione e l'altra esattamente pari alla distanza intergeofonica.

Con un registratore a 48 canali si può ottenere una copertura multipla del $1200 \%$ energizzando ogni due distanze intergeofoniche mentre se si energizza ogni distanza intergeofonica si ottiene una copertura multipla del $2400 \%$.

Allo stesso modo utilizzando un sismografo a 96 canali si ottiene una copertura multpla del $4800 \%$ energizzando ad ogni distanza intergeofonica; mentre se si energizza al doppio della distanza intergeofonica si ottiene una copertura multipla del $2400 \%$ e, infine, si può ottenere una copertura multipla del $1200 \%$ energizzando ogni 4 distanze intergeofoniche.

La prospezione sismica a riflessione dovrà indicativamente rispettare le seguenti correlazioni tra spaziatura intergeofonica della linea (da 24 canali di registrazione) e profondità ottimale dell'obiettivo di indagine (vedi Tab.1)

L'insieme dei dati acquisiti dovrà essere organicamente elaborato, mediante software dotati di alta valenza risolutiva, attuando nel modo più rigoroso le fasi sequenziali del proce- 
dimento analitico.

1. Correzioni statiche;

2. Muting;

3. Analisi spettrale;

4. Filtraggi sia nel dominio dei tempi che in quello delle frequenze con filtri variabili;

5. FK filter sia in velocità che polinomiali;

6. Analisi di velocità (Normal Move Out);

7. Deconvoluzione;

8. Stacking;

9. Correzioni statiche residue;

10. Migrazione.

Tab. 1 - Correlazioni tra spaziatura intergeofonica della linea (da 24 canali di registrazione) e profondità ottimale dell'obiettivo di indagine.

\begin{tabular}{|c|c|}
\hline $\begin{array}{c}\text { SPAZIATURA } \\
\text { GEOFONICA }\end{array}$ & $\begin{array}{c}\text { PROFONDITÀ } \\
\text { OBIETTIVO }\end{array}$ \\
\hline $0.5 \mathrm{~m}$ & $<25 \mathrm{~m}$ \\
\hline $1 \mathrm{~m}$ & $25-50 \mathrm{~m}$ \\
\hline $2.5 \mathrm{~m}$ & $50-100 \mathrm{~m}$ \\
\hline $5 \mathrm{~m}$ & $100-250 \mathrm{~m}$ \\
\hline $10 \mathrm{~m}$ & $>250 \mathrm{~m}$ \\
\hline
\end{tabular}

\subsubsection{Documentazione}

La documentazione di ciascuna indagine dovrà comprendere:

- informazioni generali (commessa, cantiere, ubicazione, data, nominativo dell'operatore);

- sismogrammi in originale su supporto magnetico o disco ottico delle registrazioni di campagna;

- $\quad$ sismosezioni dei tempi (ms) in ordinate e delle distanze (m) in ascisse della elaborazione finale di ogni linea sismica a riflessione;

- sismosezioni delle profondità $(\mathrm{m})$ in ordinate e delle distanze $(\mathrm{m})$ in ascisse della elaborazione finale di ogni linea sismica a riflessione;

- relazione conclusiva con indicate le metodologie impiegate, gli algoritmi e i criteri di calcolo ed elaborazione adottati con commenti sulle risultanze ottenute e correlazione con le informazioni di natura geologica dell'area in esame;

- documentazione fotografica.

\subsubsection{Rilievo topografico del piano completo di prospezione sismica}

Ad integrazione dell'indagine sismica dovrà essere eseguito un rilievo topografico comprendente la determinazione planoaltimetrica delle ubicazioni dei geofoni delle basi sismiche a riflessione, riferita a punti notevoli o ad elementi cartografici noti dell'area interessata. Le coordinate del rilievo topografico, dovranno essere inquadrate in un sistema di coordinate generale di progetto fornito dalla Società. Qualora quest'ultimo non risulti disponibile il suddetto rilievo sarà fornito in coordinate relative.
Tale rilievo dovrà essere corredato dai libretti di campagna dei rilievi ed informatizzazione dei dati nei formati digitali stabiliti dalla Società o dalla Direzione Lavori.

\subsection{Indagine sismica a riflessione ad onde di taglio (S)}

\subsubsection{Generalità}

L'indagine sismica a riflessione consiste nell' energizzazione del sottosuolo e nella registrazione degli arrivi delle onde di taglio (onde S) riflesse, in corrispondenza di geofoni orizzontali disposti secondo un allineamento con interassi tra i geofoni e lunghezza totale dello stendimento tali da permettere una adeguata profondità di indagine.

\subsubsection{Caratteristiche delle attrezzature}

Lattrezzatura di prova consigliata dovrà essere costituita dai seguenti componenti:

- $\quad$ sismografo minimo a 24 canali, con possibilità di stack degli impulsi sismici, filtri analogici e digitali programmabili (filtri attivi tipo high pass, band pass e band reject), guadagno verticale del segnale (in ampiezza) e sensibilità tra 6 e 92 decibel, registrazione dei dati in digitale per elaborazioni successive con formato in uscita minimo a 16 bit;

- minimo 24 geofoni orizzontali a frequenza propria variabile tra 25 e $100 \mathrm{~Hz}$;

- Sistema di energizzazione adeguato alla profondità di indagine che potrà essere costituito $\mathrm{da}$ :

- martello strumentato agente lateralmente su un blocco adeguatamente ancorato (per attrito radente) al terreno;

- energizzatori oleopneumatici e/o gravimetrici trainati e/o trasportati (pendoli) che producono onde di taglio polarizzate sul piano orizzontale;

- vibroseis.

\subsubsection{Modalità esecutive}

Il rilievo sismico a riflessione dovrà essere eseguito per mezzo di stese lineari con geofoni posti ad intervalli regolari scelti in relazione alla profondità dell'obiettivo da raggiungere. In generale l'interdistanza tra i geofoni dovrà essere pari a $0.5,1$, 2.5, 5, 10 o 20 metri.

I punti di origine dell'energia dovranno essere ubicati o nel centro di simmetria del gruppo di registrazione (metodologia "Split Spread") oppure ad un estremo dello stendimento (metodologia "End On") o infine in posizione distanziata dai due punti precedenti fino ad un massimo di 30-50 metri dalla stesa ed in direzione parallela alla stesa stessa.

L'indagine e l'elaborazione dei dati dovranno garantire una "copertura multipla" minima del $1200 \%$.

La prospezione sismica a riflessione dovrà essere realizzata secondo criteri di "stacking orizzontale" mediante copertura multipla di stendimenti in ragione variabile da un minimo del $1200 \%$ secondo gli indirizzi e la finalità dell'indagine.

Per ottenere una copertura multipla del $1200 \%$ con un sismografo da 24 canali è necessario energizzare lungo linea con 
una interdistanza fra una energizzazione e l'altra esattamente pari alla distanza intergeofonica.

Con un registratore a 48 canali si può ottenere una copertura multipla del $1200 \%$ energizzando ogni due distanze intergeofoniche mentre se si energizza ogni distanza intergeofonica si ottiene una copertura multipla del $2400 \%$.

Allo stesso modo utilizzando un sismografo a 96 canali si ottiene una copertura multpla del $4800 \%$ energizzando ad ogni distanza intergeofonica; mentre se si energizza al doppio della distanza intergeofonica si ottiene una copertura multipla del $2400 \%$ e, infine, si può ottenere una copertura multipla del $1200 \%$ energizzando ogni 4 distanze intergeofoniche.

La prospezione sismica a riflessione dovrà indicativamente rispettare le seguenti correlazioni tra spaziatura intergeofonica della linea (da 24 canali di registrazione) e profondità ottimale dell'obiettivo di indagine. (Tab. 2)

Tab. 2 - Correlazioni tra spaziatura intergeofonica della linea (da 24 canali di registrazione) e profondità ottimale dell'obiettivo di indagine.

\begin{tabular}{|c|c|}
\hline $\begin{array}{c}\text { SPAZIATURA } \\
\text { GEOFONICA }\end{array}$ & $\begin{array}{c}\text { PROFONDITÀ } \\
\text { OBIETTIVO }\end{array}$ \\
\hline $0.5 \mathrm{~m}$ & $<25 \mathrm{~m}$ \\
\hline $1 \mathrm{~m}$ & $25-50 \mathrm{~m}$ \\
\hline $2.5 \mathrm{~m}$ & $50-100 \mathrm{~m}$ \\
\hline $5 \mathrm{~m}$ & $100-250 \mathrm{~m}$ \\
\hline $10 \mathrm{~m}$ & $>250 \mathrm{~m}$ \\
\hline
\end{tabular}

L'insieme dei dati acquisiti dovrà essere organicamente elaborato, mediante software dotati di alta valenza risolutiva, attuando nel modo più rigoroso le fasi sequenziali del procedimento analitico.

1. Correzioni statiche;

2. Muting;

3. Analisi spettrale;

4. Filtraggi sia nel dominio dei tempi che in quello delle frequenze con filtri variabili;

5. FK filter sia in velocità che polinomiali;

6. Analisi di velocità (Normal Move Out);

7. Deconvoluzione;

8. Stacking;

9. Correzioni statiche residue;

10. Migrazione.

\subsubsection{Documentazione}

La documentazione di ciascuna indagine dovrà comprendere:

- informazioni generali (commessa, cantiere, ubicazione, data, nominativo dell'operatore);

- sismogrammi in originale su supporto magnetico o disco ottico delle registrazioni di campagna;

- sismosezioni dei tempi (ms) in ordinate e delle distanze $(\mathrm{m})$ in ascisse della elaborazione finale di ogni linea sismica a riflessione;
- $\quad$ sismosezioni delle profondità $(m)$ in ordinate e delle distanze $(\mathrm{m})$ in ascisse della elaborazione finale di ogni linea sismica a riflessione;

- relazione conclusiva con indicate le metodologie impiegate, gli algoritmi e i criteri di calcolo ed elaborazione adottati con commenti sulle risultanze ottenute e correlazione con le informazioni di natura geologica dell'area in esame;

- documentazione fotografica.

\subsubsection{Rilievo topografico del piano completo di prospezione sismica}

Ad integrazione dell'indagine sismica dovrà essere eseguito un rilievo topografico comprendente la determinazione planoaltimetrica delle ubicazioni dei geofoni delle basi sismiche a riflessione, riferita a punti notevoli o ad elementi cartografici noti dell'area interessata. Le coordinate del rilievo topografico, dovranno essere inquadrate in un sistema di coordinate generale di progetto fornito dalla Società. Qualora quest'ultimo non fosse disponibile il suddetto rilievo sarà fornito in coordinate relative.

Tale rilievo dovrà essere corredato dai libretti di campagna dei rilievi ed informatizzazione dei dati nei formati digitali stabiliti dalla Società o dalla Direzione Lavori.

\subsection{Prospezione sismica in foro di sondaggio (Down- Hole)}

\subsubsection{Generalità}

La prova consiste nella misurazione dei tempi di arrivo di impulsi sismici generati in superficie ad uno o più ricevitori posti all'interno di un foro di sondaggio verticale, adeguatamente rivestito con apposita tubazione in PVC o ABS con spessore $>3 \mathrm{~mm}$ da assemblare mediante filettatura M/F oppure con manicotti di giunzione incollati; tale rivestimento dovrà essere cementato al terreno incassante mediante opportuna miscela cementizia.

La prova consente la misura diretta delle velocità di propagazione $V_{P}$ delle onde di compressione (onde $P$ ) e $V_{S}$ delle onde di taglio (onde $\mathrm{SH}$ ) utili alla determinazione dei parametrici elastici dei terreni in condizioni dinamiche.

\subsubsection{Caratteristiche delle attrezzature}

Lattrezzatura di prova dovrà essere costituita dai seguenti componenti:

- $\quad$ sistema di energizzazione (per onde di compressione P) costituito da:

- martello strumentato lasciato cadere con energia su una piastra metallica appoggiata al suolo;

- cannoncino sismico;

- energizzatori oleopneumatici e/o gravimetrici trainati e/o trasportati.

- $\quad$ sistema di energizzazione (per onde di taglio $\mathrm{SH}$ ) costituito da una massa battente manovrata a mano, pneumaticamente o oleopneumaticamente agente a percussione sul piano orizzontale in modo coniugato $\left(180^{\circ}\right)$ su un'incu- 
dine di legno o di altro materiale, ben saldo al terreno solo per attrito radente e posto nelle adiacenze della testa foro;

- geofoni da foro tridimensionali, a frequenza compresa fra 8 e $14 \mathrm{~Hz}$, e di diametro minore o uguale a $70 \mathrm{~mm}$, da calare nel foro a profondità prefissate, in grado di registrare i tempi di arrivo delle onde di compressione e di taglio; ogni ricevitore deve potere essere reso solidale con la tubazione di rivestimento del foro tramite un dispositivo di bloccaggio meccanico, pneumatico e/o elettrico. In caso si utilizzi un solo ricevitore, questo potrà essere anche:

- a doppia terna ovvero costituito da due terne cartesiane ortogonali di ricevitori spaziate fra loro di un metro (1 Verticale e 2 Orizzontali);

- costituito da 3 o più geofoni orizzontali (1 Verticale 3 o più geofoni Orizzontali) disposti sul piano orizzontale ad angoli variabili $\left(60^{\circ}\right.$ se 3 geofoni orizzontali $-45^{\circ}$ se 4 geofoni orizzontali).

- sismografo registratore con un numero di canali uguale o superiore al numero di ricevitori utilizzati, in grado di realizzare campionature di segnali tra 0.025 e 2 millisecondi e dotato di filtri high pass, band pass e band reject, di "Automatic Gain Control” e di convertitori A/D del segnale campionato ad almeno 16 bit;

- apposito software per l'elaborazione dei dati, in grado di fornire i valori di velocità delle onde di compressione e di taglio per ogni stazione di misura impiegando iterativamente algoritmi di calcolo adeguati (es. ART, SIRT, e ILSP) previo controllo dei tragitti dei raggi sismici (Ray Tracing Curvilineo).

\subsubsection{Modalità esecutive}

Le modalità di esecuzione della prova dovranno essere le seguenti:

- posizionamento e bloccaggio degli energizzatori delle onde di compressione e di taglio in prossimità della bocca pozzo (a qualche metro di distanza dai $2-5 \mathrm{~m}$ ).

- posizionamento e bloccaggio del ricevitore a fondo foro;

- generazione di un impulso di taglio normale e coniugato con relativa registrazione dei tempi di arrivo delle onde di taglio per verifica dei parametri di acquisizione (record time). Durante questo test si deve riconoscere chiaramente l'arrivo delle onde di taglio mediante inversione di polarità del segnale acquisito. Stabiliti gli esatti parametri di acquisizione si procede con la registrazione nel seguente modo:

- energizzazione delle onde di compressione e registrazione del file relativo

- $\quad$ energizzazione delle onde di taglio e registrazione del file relativo

- riposizionamento del ricevitore 1 metro (o quanto stabilito dalla DL) più superficiale rispetto a fondo foro e ripetizione delle energizzazioni di compressione e di taglio come sopra
- $\quad$ ripetizione delle medesime operazioni lungo tutta la verticale d'indagine .

Le misure saranno relative all'intervallo di profondità e avranno frequenza non inferiore a 1 misura ogni metro di sondaggio.

\subsubsection{Documentazione}

La documentazione di ciascuna indagine dovrà comprendere:

- informazioni generali (commessa, cantiere, ubicazione, data, nominativo dell'operatore);

- la quota assoluta o relativa della testa della tubazione di misura;

- $\quad$ sismogrammi in originale delle registrazioni di campagna su supporto magnetico;

- relazione conclusiva, elaborata in base ai risultati delle indagini svolte in cui saranno indicati:

- $\quad$ gli algoritmi di calcolo impiegati, tabelle e tavole ad integrazione e chiarimento delle analisi;

- diagrafie riportanti:

- stratigrafia del sondaggio;

- tempi di arrivo delle onde di compressione e di taglio;

- velocità delle onde di compressione e di taglio per ogni stazione;

- velocità intervallari delle onde di compressione e di taglio;

- attenuazione e fattore qualità;

- coefficiente di Poisson dinamico;

- modulo di elasticità dinamico;

- modulo di taglio dinamico;

- modulo di compressibilità dinamico;

- tracce sismografiche onde di compressione;

- tracce sismografiche onde di taglio;

\subsection{Prospezione sismica tra fori di sondaggio (Cross- Hole)}

\subsubsection{Generalità}

La prova consiste nella misurazione dei tempi di arrivo di impulsi sismici generati in profondità all'interno di un foro di sondaggio verticale ad un ricevitore posto all'interno di un secondo foro di sondaggio verticale. I fori, paralleli e adeguatamente rivestiti con apposita tubazione, dovranno essere ad una distanza reciproca generalmente compresa tra 3 e $8 \mathrm{~m}$.

La prova consente la misura diretta delle velocità di propagazione $\mathrm{V}_{\mathrm{P}}$ delle onde di compressione (onde $\mathrm{P}$ ) e $\mathrm{V}_{\mathrm{S}}$ delle onde di taglio (onde $\mathrm{Vs}_{\mathrm{V}}$ o $\mathrm{Vs}_{\mathrm{H}}$ ) e la determinazione dei parametrici elastici dei terreni in condizioni dinamiche.

\subsubsection{Normative e specifiche di riferimento}

- ISRM -"Suggested methods for seismic testing within and between boreholes", 1988 


\subsubsection{Caratteristiche delle attrezzature}

Lattrezzatura di prova dovrà essere costituita almeno dai seguenti componenti:

- sistema di energizzazione per onde di compressione di tipo pneumatico, oleodinamico elettrico o a capsula esplodente

- N.B. l'energizzatore deve contenere al proprio interno un dispositivo di controllo del T"0" ovvero deve trasmettere al ricevitore oltre che l'impulso del T"O" anche una traccia sismografica che permetterà di eliminare gli eventuali errori del T"0" stesso. Se così non fosse sarà necessario realizzare il Cross-Hole fra tre sondaggi e non più fra due.

- Sistema di energizzazione per onde di taglio oleodinamico che possa produrre energia polarizzata ed invertibile sul piano verticale, in grado di fornire energia in quantità sufficiente alla generazione di impulsi in modo che risultino leggibili; è onere e responsabilità dell'Impresa dimensionare correttamente il sistema di energizzazione, in funzione della natura e delle caratteristiche dei terreni e che sono da considerarsi noti, in quanto le misure sono successive alla perforazione dei sondaggi entro i quali si eseguono le stesse. N.B. l'energizzatore deve contenere al proprio interno un dispositivo di controllo del T"0" ovvero deve trasmettere al ricevitore oltre che l'impulso del T"0" anche una traccia sismografica che permetterà di eliminare gli eventuali errori del T"0" stesso. Se così non fosse sarà necessario realizzare il Cross-Hole fra tre sondaggi e non più fra due.

- Uno o più geofoni da foro tridimensionali, a frequenza compresa fra 8 e $14 \mathrm{~Hz}$, di diametro minore o uguale a $70 \mathrm{~mm}$, da calare nel foro a profondità prefissate, in grado di registrare i tempi di arrivo delle onde di compressione e di taglio; ogni ricevitore deve potere essere reso solidale con la tubazione di rivestimento del foro tramite un dispositivo di bloccaggio meccanico, pneumatico e/o elettrico;

- sismografo registratore con un numero di canali uguale o superiore al numero di ricevitori utilizzati, in grado di realizzare campionature di segnali tra 0.025 e 2 millesecondi e dotato di filtri high pass, band pass e band reject, di "Automatic Gain Control" e di convertitori A/D del segnale campionato ad almeno 16 bit;

- apposito software per l'elaborazione dei dati, in grado di fornire i valori di velocità delle onde di compressione e di taglio per ogni stazione di misura impiegando iterativamente algoritmi di calcolo adeguati (es. ART, SIRT, e ILSP), previo controllo dei tragitti dei raggi sismici (Ray Tracing Curvilineo).

\subsubsection{Modalità esecutive}

Le modalità di esecuzione della prova dovranno essere le seguenti:

- posizionamento e bloccaggio di sorgente (in un foro) e ricevitore (nell'altro foro) in corrispondenza della prima coppia di posizioni coniugate, in accordo con il progetto delle indagini; sorgente e ricevitore dovranno essere posizionati alla medesima profondità, in modo da realizzare un percorso delle onde ipoteticamente orizzontale;

- generazione dell'impulso (è ammessa anche la somma di più impulsi) e registrazione dei tempi di arrivo delle onde di compressione;

- generazione dell'impulso (è ammessa anche la somma di più impulsi) e registrazione dei tempi di arrivo delle onde di taglio polarizzate sul piano verticale in direzione destra (o sinistra);

- generazione dell'impulso (è ammessa anche la somma di più impulsi) e registrazione dei tempi di arrivo delle onde di taglio polarizzate sul piano verticale in direzione sinistra (o destra);

- $\quad$ ripetizione delle medesime operazioni per ciascuna coppia di punti coniugati lungo le due verticali d'indagine.

Le misure saranno relative all'intervallo di profondità e avranno frequenza stabilita dalla DL (solitamente 1 misura ogni metro).

È necessario altresì procedere con la misura della verticalità dei sondaggi stessi ovvero bisogna stabilire esattamente la distanza reciproca esistente fra i due (o più) sondaggi alle varie quote di misura delle onde di compressione e di taglio.

Per queste misure verrà utilizzata una sonda inclinometrica con 2 sensori ortogonali con sensibilità non inferiore a 0.1 gradi. La sonda sarà del tipo a controllo azimutale o sarà calata con aste con connessione a baionetta, in grado di evitare modifiche dell'orientazione azimutale della sonda per l'intera profondità. Le misure verranno effettuate ogni $1 \div 2 \mathrm{~m}$ e la loro restituzione grafica, che evidenzi la distanza in ogni punto della coppia di fori cross-hole, farà parte integrante della documentazione.

\subsubsection{Documentazione}

La documentazione di ciascuna indagine dovrà comprendere:

- informazioni generali (commessa, cantiere, ubicazione, data, nominativo dell'operatore);

- le modalità esecutive del foro;

- lo schema geometrico di ogni tubazione installata;

- la quota assoluta o relativa della testa della tubazione di misura;

- le caratteristiche della tubazione installata;

- modalità di iniezione, quantità e composizione della miscela iniettata nell'intercapedine;

- grafici e tabulati del rilievo della verticalità presentati in modo che risulti facilmente determinabile la distanza fra i fori alle diverse profondità;

- $\quad$ sismogrammi in originale delle registrazioni di campagna su supporto magnetico;

- relazione conclusiva, elaborata in base ai risultati delle indagini svolte in cui saranno indicati:

- gli algoritmi di calcolo impiegati, tabelle e tavole ad integrazione e chiarimento delle analisi; 
- diagrafie riportanti:

- stratigrafie dei sondaggi;

- tempi di arrivo delle onde di compressione e di taglio;

- velocità delle onde di compressione e di taglio nella sezione compresa tra i due fori;

- coefficiente di Poisson dinamico;

- modulo di elasticità dinamico;

- modulo di taglio dinamico;

- modulo di compressibilità dinamico;

- tracce sismografiche onde di compressione;

- tracce sismografiche onde di taglio;

- misure clinometriche (della verticalità dei fori di sondaggio);

- risultanze finali ed interpretative.

\subsection{Prospezione sismica con cono sismico}

\subsubsection{Generalità}

La prova consiste nella misurazione dei tempi di arrivo di impulsi sismici di taglio (SH) generati in superficie ad un ricevitore posto all'interno di un' asta penetrometrica opportunamente attrezzata con una punta sismica.

La prova consente la misura diretta delle velocità di propagazione $\mathrm{Vs}_{\mathrm{H}}$ delle onde di taglio (onde $\mathrm{SH}$ ) fra due ricevitori posti all'interno si una punta sismica applicata ad un penetrometro.

\subsubsection{Caratteristiche delle attrezzature}

Lattrezzatura di prova dovrà essere costituita almeno dai seguenti componenti:

- $\quad$ sistema di energizzazione (per onde di taglio SH) costituito da una massa battente manovrata a mano, pneumaticamente o oleopneumaticamente agente a percussione sul piano orizzontale in modo coniugato $\left(180^{\circ}\right)$ su un'incudine di legno o di altro materiale, ben saldo al terreno solo per attrito radente e posto nelle adiacenze della testa foro;

- Punta penetrometrica sismica costituita da un corpo metallico e da due ricevitori sismici (geofoni e/o accelerometri) paralleli fra loro a distanza di 1 metro l'uno dall'altro incapsulati ed opportunamente isolati nella punta sismica;

- sismografo registratore con un numero di canali uguale o superiore al numero di ricevitori utilizzati in grado di realizzare campionature di segnali tra 0.025 e 2 millisecondi e dotato di filtri high pass, band pass e band reject, di "Automatic Gain Control" e di convertitori A/D del segnale campionato ad almeno 16 bit.

\subsubsection{Modalità esecutive}

Le modalità di esecuzione della prova dovranno essere le seguenti:

- posizionamento e bloccaggio degli energizzatori delle onde di taglio in prossimità della prova penetrometrica da realizzare $(2-5 \mathrm{~m})$;

- Infilaggio della punta sismica nel terreno fino alla posi- zione in cui si avrà il primo ricevitore a $-1 \mathrm{~m}$ dal p.c. e il secondo al p.c.; la direzione dei ricevitori dovrà essere parallela alla direzione di polarizzazione dell'energizzatore;

- Impostare i parametri di registrazione del sismografo in modo tale che l'intervallo di campionamento dello stesso sia posizionato sul valore massimo e il tempo di registrazione sia di almeno 300 millisecondi;

- generazione di un impulso di taglio in una direzione (es normale) e registrazione dei tempi di arrivo dell' onda di taglio;

- generazione di un impulso di taglio nella direzione coniugata $\left(180^{\circ}\right.$ sul piano orizzontale) e registrazione dei tempi di arrivo dell'onda di taglio;

- Infilaggio della punta penetrometrica 1 metro più in profondità senza far ruotare le aste. In tal modo l'intervallo di ricezione sarà fra -2 e $-1 \mathrm{~m}$ dal p.c. e si ripetono le energizzazioni fino al rifiuto e / o fino alla profondità richiesta.

\subsubsection{Documentazione}

La documentazione di ciascuna indagine dovrà comprendere:

- informazioni generali (commessa, cantiere, ubicazione, data, nominativo dell'operatore);

sismogrammi in originale delle registrazioni di campagna su supporto magnetico;

- relazione conclusiva, elaborata in base ai risultati delle indagini svolte in cui saranno indicati:

- gli algoritmi di calcolo impiegati, tabelle e tavole ad integrazione e chiarimento delle analisi;

- diagrafie riportanti:

- tempi di arrivo delle onde di taglio;

- velocità intervallari delle onde di taglio;

- tracce sismografiche onde di taglio.

\subsection{Prospezione Geotomografica (fra sondaggi o fra sondaggio e superficie topografica)}

\subsubsection{Generalità}

Nella prospezione sismica geotomografica, eseguita in corrispondenza di una sezione delimitata da due superfici comunque inclinate (costituite ad es. da due fori di sondaggio oppure dalla superficie topografica e un foro di sondaggio) si utilizza una superficie come superficie di energizzazione e l'altra come superficie di ricezione.

Nel caso in cui ci si trovi di fronte a una prospezione fra due sondaggi in un sondaggio saranno posizionati dei ricevitori e nell'altro verranno effettuate le energizzazioni.

$\mathrm{Nel}$ caso invece in cui ci trovi di fronte a una prospezione fra un sondaggio e la superficie topografica saranno posizionati nel sondaggio i ricevitori e sulla superficie topografica verranno effettuate le energizzazioni (eventualmente si possono invertire le posizioni)

\subsubsection{Caratteristiche delle attrezzature}

Lattrezzatura di prova dovrà essere costituita almeno dai seguenti componenti: 
- sismografo a 24 canali, con possibilità di stack degli impulsi sismici, filtri analogici e digitali programmabili (filtri attivi tipo high pass, band pass e band reject), guadagno verticale del segnale (in ampiezza) e sensibilità tra 6 e 92 decibel, registrazione dei dati in digitale per elaborazioni successive con formato in uscita minimo a 16 bit;

- sistema di energizzazione per onde di compressione di tipo pneumatico, oleodinamico elettrico o a capsula esplodente (per le energizzazioni in pozzo);

- geofoni di superficie a frequenza propria variabile tra 8 e $100 \mathrm{~Hz}$ (per le ricezioni sulla superficie topografica);

- geofoni da pozzo o idrofoni (se in falda), a frequenza propria variabile tra 8 e $14 \mathrm{~Hz}$ (per le ricezioni in pozzo);

- $\quad$ sistema di energizzazione per le onde di compressione da superficie che può essere costituito da:

- martello strumentato lasciato cadere con violenza su una piastra metallica appoggiata al suolo;

- cannoncino sismico;

- energizzatori oleopneumatici e/o gravimetrici trainati e/o trasportati.

\subsubsection{Modalità esecutive \\ -Fra due sondaggi}

Tali indagini vengono effettuate, solitamente, per la determinazione e la definizione di oggetti sepolti all'interno di un terreno incassante sia il caso di cavità o di fondazioni in jet grouting e/o pali di fondazioni nonché per la definizione di eventuali piani di fatturazione tettonica nello spazio compreso fra le due verticali.

La prospezione sarà tanto più precisa e definita quanto più le superfici di indagine saranno vicine fra loro e la distanza fra i ricevitori/emissioni di energia sarà piccola.

In linea di massima la distanza fra i ricevitori/emissioni di energia sarà non più vicini di 0.5 metri e la distanza minima fra i sondaggi non dovrà essere superiore ai 10 metri

Solitamente si utilizzano stringhe di 24 ricevitori in pozzo spaziati 0,5 metri e si emettono impulsi di energia nel pozzo adiacente ad intervalli di $0.5 \mathrm{~m}$ alle stesse quote dei ricevitori.

\section{-Fra sondaggio e superficie topografica}

Tali indagini vengono effettuate, solitamente, per la determinazione di cavità o per la definizione di eventuali piani di fatturazione tettonica o superfici di allentamento della scarpata.

Solitamente la superficie topografica è molto inclinata (scarpata) rispetto all'orizzontale e il sondaggio è realizzato a bordo scarpata.

Tale prospezione viene realizzata inserendo di solito nel sondaggio una stringa di 24 ricevitori interspaziati $0,5 \mathrm{~m}$ e si energizza lungo la scarpata a distanza di 0.5 metri (in quota).

Sarà altresì possibile posizionare 24 ricevitori lungo la scarpata ed energizzare nel sondaggio per 24 volte alla stessa quota dei ricevitori.

\subsubsection{Elaborazione dei dati}

L'elaborazione dovrà essere sviluppata tramite un'analisi con modellazione del sottosuolo su base anisotropica, la quale dovrà fornire, previa elaborazione con metodologie iterative R.T.C. (Ray Tracing Curvilineo) e algoritmi di ricostruzione tomografica (ad esempio con l'impiego di algoritmi ART - Algebric Reconstruction Technique, SIRT - Simultaneous Iterative Reconstruction Technique o ILST - Iterative Least Square Technique), il campo delle velocità del sottosuolo ad elevata densità di informazioni: le celle unitarie, di forma rettangolare, potranno avere dimensioni orizzontali (asse $\mathrm{x}$ ) e verticali (asse z) pari a, rispettivamente, $1 / 3 \div 1 / 5$ e $1 / 5 \div 1 / 10$ della spaziatura tra i geofoni.

\subsubsection{Documentazione}

La documentazione di ciascuna indagine dovrà comprendere:

- informazioni generali (commessa, cantiere, ubicazione, data, nominativo dell'operatore);

- sismogrammi originali su supporto magnetico;

- mappature in tonalità di colore relative alla sezione tomografica in termini di ray tracing, di densità dei dati e di velocità delle onde di compressione $\left(\mathrm{V}_{\mathrm{P}}\right)$;

- tabelle con i parametri di input e calcolati;

- relazione in cui vengono riportate le metodologie di indagine, gli algoritmi impiegati, le analisi realizzate e le risultanze dell'analisi.

\subsubsection{Rilievo topografico del piano completo di prospezione sismica}

Ad integrazione dell'indagine sismica dovrà essere eseguito un rilievo topografico comprendente la determinazione planoaltimetrica delle ubicazioni dei ricevitori/sorgenti lungo la scarpata, riferita a punti notevoli (bocca pozzo) o ad elementi cartografici noti dell'area interessata. Le coordinate del rilievo topografico, dovranno essere inquadrate in un sistema di coordinate generale di progetto fornito dalla Società. Qualora quest'ultimo non fosse disponibile il suddetto rilievo sarà fornito in coordinate relative.

Tale rilievo dovrà essere corredato dai libretti di campagna dei rilievi ed informatizzazione dei dati nei formati digitali stabiliti dalla Società o dalla Direzione Lavori.

È necessario altresì procedere con la misura della verticalità dei sondaggi stessi ovvero bisogna stabilire esattamente la distanza reciproca esistente fra i sondaggi (o il sondaggio) alle varie quote di misura delle onde di compressione.

Per queste misure verrà utilizzata una sonda inclinometrica con 2 sensori ortogonali con sensibilità superiore a 0.07 gradi. La sonda sarà del tipo a controllo azimutale o sarà calata con aste con connessione a baionetta, in grado di evitare modifiche dell'orientazione azimutale della sonda per l'intera profondità. Le misure verranno effettuate ogni $1 \div 2 \mathrm{~m}$ e la loro restituzione grafica, che evidenzi la distanza in ogni punto della coppia di fori cross-hole, farà parte integrante della documentazione. 


\subsection{Prove per onde superficiali attive}

\subsubsection{Generalità}

Il metodo delle onde superficiali è una tecnica di caratterizzazione sismica basata sull'analisi della dispersione geometrica delle onde superficiali (solitamente si tratta di onde di Rayleigh in caso di acquisizioni terrestri, ma possono essere utilizzate anche le onde di Love). In particolare, poiché le onde di Rayleigh si propagano lungo la superficie topografica in uno strato di profondità circa pari alla lunghezza d'onda, in mezzi stratificati verticalmente eterogenei, le diverse lunghezze d'onda, relative alla propagazione di armoniche a diversa frequenza, si propagano con velocità di fase che dipende dalle proprietà dei materiali interessati dalla propagazione. Ciò fa sì che la velocità di fase sia funzione della frequenza dell'onda stessa. Tale dipendenza prende il nome di dispersione geometrica e viene rappresentata tramite la curva di dispersione (velocità di fase vs. frequenza). La propagazione delle onde di superficie è un fenomeno multimodale, una stessa frequenza può propagarsi contemporaneamente a diverse velocità, e ogni velocità viene chiamata modo.

Dalla curva di dispersione sperimentale è possibile caratterizzare il sottosuolo in termini di variazione della velocità di propagazione delle onde di taglio $\left(\mathrm{V}_{\mathrm{SV}}\right)$, e quindi del modulo di rigidezza al taglio dinamico $\left(\mathrm{G}_{0}\right)$, in funzione della profondità.

Lutilizzo delle prove per onde superficiali ha molti campi di applicazione, dalla stima della $\mathrm{V}_{\mathrm{S} 30}$ richiesta dalle NTC 08, alla valutazione dei parametri utili a studi approfonditi di pericolosità sismica; dalla caratterizzazione di discariche, alla valutazione della profondità e dello stato di alterazione di substrati superficiali in presenza di falde acquifere, la caratterizzazione di versanti e depositi.

Generalmente il metodo assume come modello del terreno un mezzo elastico-lineare a strati omogenei. Nella maggior parte delle applicazioni, la tecnica è quindi utilizzata per stimare un profilo verticale 1D di Vs, ma vi sono molte applicazioni in siti con moderate variabilità laterali in cui il metodo consente di stimare distribuzioni di Vs pseudo-2D/3D.

Il metodo per onde superficiali maggiormente utilizzato prende comunemente il nome di MASW (Multichannel Analysis of Surface Waves) e si basa sulla misura delle onde superficiali eseguita con un dispositivo lineare di sensori sismici e con sorgenti artificiali.

\subsubsection{Caratteristiche delle attrezzature}

Lattrezzatura di prova dovrà essere costituita almeno dai seguenti componenti:

- sismografo digitale a 12 canali (meglio 24), con possibilità di stack delle registrazioni, guadagno del segnale (in ampiezza) e dinamica del convertitore $\mathrm{A} / \mathrm{D}$ minima a 16 bit;

- per prove relative alla caratterizzazione del sottosuolo, 12 (meglio 24) geofoni verticali (o accelerometri) a frequenza propria uguale o inferiore a $4.5 \mathrm{~Hz}$; per prove non distruttive su pavimentazioni o manufatti è possibile uti- lizzare sensori a frequenza maggiore;

- sistema di energizzazione costituito da uno dei seguenti dispositivi da scegliersi in funzione della scala delle indagini:

- massa battente con eventuale piastra di ripartizione appoggiata al suolo;

- energizzatori sismici impulsivi oleopneumatici e/o a gravità;

- fucili sismici;

- cariche di esplosivo;

- sorgenti vibranti (vibrodina).

\subsubsection{Modalità esecutive}

La procedura consiste in tre step principali: l'acquisizione dei dati sismici sul terreno; l'elaborazione per la stima delle curve di dispersione sperimentali e l'inversione delle curve di dispersione, volta alla stima dei profili di $\mathrm{V}_{\mathrm{S}}$ che costituisce il risultato della prova.

\subsubsection{Acquisizione}

La fase di acquisizione, prevede l'utilizzo di una sorgente (impulsiva o controllata) tramite la quale creare una perturbazione sismica che si propaga lungo la superficie libera che viene rilevata da più ricevitori (di norma geofoni verticali a bassa frequenza) posti lungo dispositivi lineari sul piano campagna. La sorgente viene posta ad un estremo dello stendimento di misura e di norma si effettuano energizzazioni ai due lati opposti dello stendimento per confrontare i risultati (si suggerisce di effettuare almeno 10 ripetizioni dell'energizzazione - con eventuale stacking - per ogni punto sorgente). Gli obiettivi dell'acquisizione sono quelli di fornire dati relativi alla propagazione di onde superficiali in una banda di frequenza più ampia possibile poiché ciò consente di ottenere informazioni sulle proprietà degli strati profondi, che influenzano le componenti a bassa frequenza, e di descrivere con adeguata risoluzione gli strati più superficiali, che influenzano in maniera significativa soprattutto le componenti ad alta frequenza. Ciò implica la scelta di corretti parametri di acquisizione nello spazio e nel tempo.

Il campionamento temporale deve garantire che tutto il treno d'onda relativo alla propagazione delle onde superficiali sia registrato, quindi la durata dell'acquisizione (T) deve essere maggiore o uguale al tempo necessario per l'armonica più lenta a raggiungere il sensore più lontano. L'intervallo di campionamento $(\mathrm{dt})$ deve garantire la stima delle armoniche di interesse in base alle regole del campionamento (frequenza di campionamento almeno doppia (meglio pari a cinque volte) della frequenza più alta da registrare).

Il campionamento spaziale deve consentire di stimare affidabilmente le lunghezze d'onda di interesse. In particolare la lunghezza dello stendimento di misura $(\mathrm{L})$ non deve essere inferiore alla metà della massima lunghezza d'onda e la distanza intergeofonica $(\mathrm{dl})$ deve essere metà della minima lunghezza d'onda.

Inoltre, poiché dal punto di vista fisico, le onde di superficie sono sensibili alle proprietà del mezzo in cui si propagano fino a circa $\lambda / 2.5-\lambda / 3$ la lunghezza $L$ deve essere definita anche in 
Tab. 3 - Parametri di acquisizione per indagini a scala ingegneristica.

\begin{tabular}{|c|c|c|c|c|}
\hline $\begin{array}{c}\text { Profondità } \\
\text { d'indagine }\end{array}$ & $\begin{array}{c}\mathrm{T} \\
{[\mathrm{s}]}\end{array}$ & $\begin{array}{c}\mathrm{dt} \\
{[\mathrm{ms}]}\end{array}$ & $\begin{array}{c}\mathrm{L} \\
{[\mathrm{m}]}\end{array}$ & $\begin{array}{c}\mathrm{dl} \\
{[\mathrm{m}]}\end{array}$ \\
\hline 1 (pavimentazioni) & 0,15 & 0.125 & 3 & 0.1 \\
\hline 10 & 1 & 0.5 & 23 & 1 \\
\hline 30 & 2 & 1 & 46 & 2 \\
\hline 100 & 4 & 2 & 200 & $5-10$ \\
\hline
\end{tabular}

funzione della profondità d'indagine desiderata.

Tipicamente per indagini a scala ingegneristica sono da considerarsi adeguati, in senso cautelativo, i parametri di acquisizione indicati in tabella 3.

I parametri di acquisizione precedentemente definiti per un profilo di misura singolo, possono anche essere adottati per l'acquisizione in modalità "continuous profiling" che prevede di traslare tutto il dispositivo di misura lungo una linea di acquisizione per stimare una serie di curve di dispersione.

$\mathrm{Nel}$ caso in cui dalle registrazioni sismiche effettuate per l'analisi delle onde superficiali si vogliano anche stimare i tempi di primo arrivo da utilizzare in indagini sismiche a rifrazione, $\mathrm{i}$ parametri indicati dovranno essere adeguati a soddisfare i requisiti di entrambe le tecniche.

\subsubsection{Elaborazione dei dati}

Esistono diverse tecniche di processing per stimare le curve di dispersione sperimentali a partire dai sismogrammi registrati. Le metodologie più diffuse sono l'analisi spettrale in dominio f-k (frequenza-numero d'onda) e $\omega$-p (frequenza angolare-lentezza) dove i massimi di energia dello spettro sono associabili alle onde di Rayleigh e vengono identificati e trasformati in punti della curva di dispersione. Gli obiettivi dell'elaborazione dei dati sono quelli di riconoscere gli eventi dispersivi e di estrarli dalla globalità dei dati isolando eventi coerenti in ampi range di frequenza e, possibilmente, riconoscendo diversi eventi associabili a diversi modi di propagazione. L'obiettivo minimo dell'elaborazione è comunque l'identificazione del modo fondamentale privo di interferenze relative ai modi superiori e altri eventi sismici (onde di volume o guidate). Quest'ultimo punto è di fondamentale importanza poiché la non corretta identificazione di un modo può portare a errori grossolani nella successiva fase di inversione. $\mathrm{E}$ opportuno che il riconoscimento dei massimi spettrali avvenga con l'ausilio di codici di ricerca automatica.

Qualora non si eseguano le operazioni di stack in acquisizione, le registrazioni relative alle diverse energizzazioni relative al medesimo punto sorgente, possono essere processati separatamente. Per ogni registrazione viene condotta la procedura di elaborazione precedentemente descritta e i risultati (velocità di fase ad ogni frequenza) possono essere trattati statisticamente per ottenere una curva di dispersione media e le incertezze ad essa associate.

È inoltre utile confrontare le curve di dispersione stimate in diversi punti sorgente per valutare l'eventuale presenza di forti discontinuità laterali.

\subsubsection{Inversione}

La curva di dispersione sperimentale (eventualmente costituita da più modi), viene utilizzata per un processo di inversione che fornisce come risultato finale il profilo verticale di $\mathrm{V}_{\mathrm{S}}$. La risoluzione del problema inverso implica la parametrizzazione del modello di sottosuolo assunto, che viene di norma schematizzato come un mezzo elastico a strati piano-paralleli, omogenei ed isotropi, nel quale l'eterogeneità è rappresentata dalla differenziazione delle caratteristiche meccaniche degli strati. I parametri di modello sono $\mathrm{V}_{\mathrm{S}}, \mathrm{V}_{\mathrm{P}}$ (o rapporto di Poisson), spessore e densità di ogni strato. I parametri incogniti dell'inversione sono $\mathrm{V}_{\mathrm{S}}$ ed eventualmente lo spessore degli strati, mentre $\mathrm{V}_{\mathrm{P}}$ (Poisson) e la densità sono assunti a priori. Inoltre, l'utilizzo di un modello monodimensionale è un'ipotesi che deve essere in linea di massima soddisfatta dal sito per garantire la validità del risultato finale.

Il processo di inversione è iterativo: a partire da un profilo di primo tentativo, costruito sulla base di metodi semplificati, ed eventualmente delle informazioni note a priori riguardo la stratigrafia, il problema diretto (simulazione della curva di dispersione teorica) viene risolto diverse volte variando i parametri incogniti di modello in modo da minimizzare lo scarto fra il set di dati sperimentali (curva di dispersione misurata) e il set di dati calcolati (curva di dispersione simulata). Il processo termina quando viene individuato un insieme di parametri di modello che renda lo scarto accettabile. Il numero dei parametri di modello deve essere scelto come il minimo in grado di descrivere la curva di dispersione sperimentale (solitamente 2-3-4 strati). Una sovraparametrizzazione rischia, infatti, di ridurre la risoluzione dei singoli parametri dovuta a problemi di equivalenza.

La procedura di inversione può essere condotta con tecniche di ricerca locale (metodi linearizzati) o globale (metodi Monte Carlo) della soluzione.

\subsubsection{Documentazione}

La relazione in cui vengono riportate le metodologie di indagine, gli algoritmi impiegati, le analisi realizzate e i risultati ottenuti dovrà contenere:

- informazioni generali (commessa, cantiere, ubicazione possibilmente georeferenziata, data, nominativo dell'operatore, parametri di acquisizione e strumentazione utilizzata);

- sismogrammi originali su supporto magnetico;

- immagini relative alla trasformata bidimensionale del campo d'onda (f-k, w-p, f-v) con massimi spettrali evidenziati;

- confronto tra la curva stimata e quella sintetica generata a partire dal modello finale di $\mathrm{V}_{\mathrm{S}}$;

- confronto tra il profilo di $\mathrm{V}_{\mathrm{S}}$ di primo tentativo e quello finale del processo di inversione;

- tabelle con i parametri calcolati e parametri assunti a priori (spessore degli strati, $\mathrm{V}_{\mathrm{S}}$, rapporto di Poisson o $\mathrm{V}_{\mathrm{P}}$ e densità). 


\subsubsection{Rilievo topografico del piano completo di prospezione sismica}

Come descritto precedentemente, per l'interpretazione delle misure di campagna si assume come modello del terreno un mezzo elastico-lineare a strati omogenei. Tale modello implica la necessità di avere un piano campagna sostanzialmente libero da ostacoli e privo di marcate irregolarità topografiche. Inoltre, per ottenere risultati significativi, è necessario che le condizioni geologiche del sottosuolo siano tali da soddisfare il modello di stratificazione sub-orizzontale.

\subsection{Prove per onde superficiali passive}

\subsubsection{Generalità}

Tali misurazioni sono in linea di principio analoghe alle prove per onde di Rayleigh con sorgente attiva. Lunica differenza è costituita dalla procedura di acquisizione e di stima della curva di dispersione sperimentale. Infatti, le misure sismiche passive non richiedono una sorgente artificiale ma sono basate sulla registrazione del rumore ambientale, che consiste in vibrazioni del terreno indotte da attività antropiche (come il traffico, il rumore derivante da macchinari industriali, ecc.), o da fenomeni naturali; tali vibrazioni del terreno prendono il nome di microtremori. Il vantaggio principale delle tecniche passive, rispetto a quelle attive, è costituito dalla possibilità di ottenere informazioni relative alla propagazione di onde a bassa frequenza e quindi di estendere le informazioni estraibili dalla curva di dispersione a profondità elevate.

L'integrazione di dati ottenuti con misure attive e passive consente, in linea teorica, di estendere l'intervallo di frequenza in cui è possibile stimare la curva di dispersione e, di conseguenza, di incrementare la profondità d'indagine raggiungibile senza perdere le informazioni di maggior dettaglio sugli strati superficiali.

\subsubsection{Caratteristiche delle attrezzature}

Lattrezzatura di prova dovrà essere costituita almeno dai seguenti componenti:

- sismografo digitale a 12 canali (meglio 24), guadagno del segnale (in ampiezza) e dinamica del convertitore A/D minima a 16 bit, possibilità di registrazione di finestre temporali di alcuni minuti;

- 4 geofoni verticali/triassiali (o accelerometri) a frequenza propria uguale o inferiore a $2 \mathrm{~Hz}$ (requisito minimo, ma si consiglia l'utilizzo di 12 ricevitori);

\subsubsection{Modalità esecutive}

Il metodo si basa sull'assunzione che il rumore sismico sia costituito prevalentemente da onde superficiali e che sia generato da sorgenti lontane dal sito di misura. Un aspetto di particolare rilevanza è costituito dalla necessità di acquisire $i$ segnali utilizzando una configurazione di prova bidimensionale sul piano campagna. Infatti, non essendo nota a priori la direzione di propagazione (funzione della posizione della sorgente che genera il rumore ambientale), è necessario eseguire una scansione spaziale che consenta di determinare la direzione di propagazione dell'onda dominante e conseguentemente la sua velocità di propagazione. Anche le tecniche di processing dovranno essere in tal senso adeguate.

\subsubsection{Acquisizione}

La strumentazione necessaria è del tutto analoga a quella utilizzata per le misure di tipo attivo; infatti si utilizzano come ricevitori dei geofoni verticali o triassiali a bassa frequenza, disposti secondo dispositivi bidimensionali, ad esempio a "L", a "T", a croce o circolari. Le geometrie dotate di un asse di simmetria verticale sono preferibili in quanto dotate della medesima risposta a tutte le possibili direzioni di provenienza del campo d'onde.

È fondamentale considerare le caratteristiche geometriche dell'array di acquisizione. Il campionamento spaziale deve consentire di stimare affidabilmente le lunghezze d'onda di interesse. Le regole del campionamento impongono che si possa stimare affidabilmente una lunghezza d'onda $(\lambda)$ massima pari a due volte la massima distanza tra ricevitori lungo la direzione di provenienza del segnale (D), mentre la minima distanza intergeofonica (d) deve essere metà della minima lunghezza d'onda misurabile.

Trattandosi di una registrazione di rumore ambientale (quindi ignota e variabile nel tempo) conviene acquisire per il maggior tempo ( $\mathrm{T}$ ) possibile consentito dal sismografo, ed eventualmente ripetere l'acquisizione per due-tre volte. L'intervallo di campionamento (dt) condiziona la massima frequenza misurabile.

Inoltre, poiché dal punto di vista fisico, le onde di superficie sono sensibili alle proprietà del mezzo in cui si propagano fino a circa $\lambda / 2.5-\lambda / 3$ la lunghezza $D$ deve essere definita anche in funzione della profondità d'indagine desiderata. Ad esempio, se si dispone di un array circolare del diametro di $100 \mathrm{~m}$, si potrà stimare affidabilmente una $\lambda$ di $200 \mathrm{~m}$ con una profondità di indagine di circa $80 \mathrm{~m}$. Ovviamente, trattandosi di misure passive, occorre che la componente in frequenza con quella lunghezza d'onda sia contenuta nel rumore ambientale. Ipotizzando che questa viaggi in un mezzo con velocità $\mathrm{di}$ fase di $1000 \mathrm{~m} / \mathrm{s}$, corrispondente ad una frequenza di $5 \mathrm{~Hz}$, tale frequenza dovrà essere una componente del rumore ambientale.

\subsubsection{Elaborazione dei dati}

Esistono diverse tecniche per acquisire ed elaborare i dati di microtremori allo scopo di ottenere una curva di dispersione, basate sull'analisi dello spettro f-k e sulla correlazione spaziale SPAC e ESAC. Esistono poi tecniche che prevedono acquisizioni passive con dispositivi monodimensionali note con la sigla RE.MI. (REfraction MIcrotremor), per cui si rimanda alla sezione 2.11.6.

I metodi basati sullo spettro $\mathrm{f}-\mathrm{k}$, ad esempio, consentono la stima dello spettro, secondo numeri d'onda in direzione qualsiasi nello spazio bidimensionale del piano campagna. Per ogni frequenza è quindi possibile ottenere uno spettro bidimensionale in dominio di numero d'onda ( $\mathrm{kx}$ e ky) e individuare in tale spettro i massimi di energia. La posizione 
del massimo spettrale a ciascuna frequenza consente la stima della direzione di arrivo dell'onda e della sua velocità, infatti, il numero d'onda associato all'onda dominante è dato dalla distanza tra il picco e l'origine degli assi. L'analisi viene ripetuta per diverse frequenze in modo da poter stimare la velocità di fase su un intervallo di frequenze sufficientemente ampio. Tale approccio consente quindi di estrarre i punti della curva di dispersione alle diverse frequenze superando il problema dell'impossibilità di conoscere le sorgenti che hanno generato il rumore misurato. Di norma, le acquisizioni durano alcuni minuti ma, in fase di elaborazione, i sismogrammi possono essere frazionati in sotto-finestre di durata più limitata. Per ogni sottofinestra viene condotta la procedura di elaborazione precedentemente descritta e i risultati (velocità di fase ad ogni frequenza) ottenuti dall'elaborazione delle diverse sotto-finestre temporali possono essere trattati statisticamente per ottenere una curva di dispersione media e le incertezze ad essa associate.

I metodi SPAC ed ESAC sono invece basati sull'autocorrelazione spaziale per la stima delle curve di dispersione. Per una particolare frequenza, la velocità di fase è uguale a quella che fa sì che l'autocorrelazione spaziale stimata descriva meglio la funzione di Bessel del primo ordine. Dal punto di vista pratico, la stima avviene confrontando queste quantità su ampi intervalli di frequenza e velocità. La curva di dispersione è rappresentata dai valori di velocità e frequenza in cui il confronto presenta il minor scarto. I metodi basati sull'autocorrelazione spaziale si fondano sull'ipotesi di omnidirezionalità del rumore.

Dal processing si ottiene dunque una curva di dispersione del tutto simile a quella che si ricava dall'interpretazione dei dati delle indagini sismiche con onde superficiali col metodo attivo che però riguarda di norma le basse frequenze.

\subsubsection{RE.MI (Refraction Microtremor)}

Il termine Refraction Microtremor non deriva dal principio fisico su cui è basato il metodo, ma dalla possibilità di utilizzare stendimenti lineari, normalmente utilizzati in indagini di sismica a rifrazione in onde P. Prima di passare alla descrizione delle modalità operative del metodo è necessario sottolineare che tale metodo è considerato in maniera molto controversa dalla comunità scientifica e che la sua adozione non è consigliata. Ciononostante tale metodo è talvolta divenuto prassi professionale soprattutto in ambiente urbano. Qualora tale approccio sia applicato, si raccomanda comunque fortemente di associarlo a misure attive.

Trattandosi di una tecnica passiva, quindi pensata per estendere la stima delle curve di dispersione a una frequenza più bassa, per le stesse ragioni di cui sopra, dovrebbe essere impiegata utilizzando sensori a frequenza propria inferiore o uguale a $4.5 \mathrm{~Hz}$ ed andrebbero adottati tempi di acquisizione lunghi con registrazioni ripetute (maggior tempo possibile consentito dal sismografo). Si sottolinea che ciò è in contrasto con le raccomandazioni tecniche indicate talvolta a livello commerciale.

A differenza delle tecniche basate sull'analisi dello spettro $\mathrm{f}-\mathrm{k}$, in questo caso è assolutamente necessario soddisfare la condizione di "omnidirezionalità" delle sorgenti, cioè si suppone che il rumore ambientale provenga sostanzialmente da tutte le direzioni. Tale assunzione è raramente verificata nella realtà.

Come per le prove per onde superficiali di tipo attivo, le metodologie più diffuse per l'elaborazione sono l'analisi spettrale in dominio $\mathrm{f}-\mathrm{k}$ (frequenza-numero d'onda) e $\omega$-p (frequenza angolare-lentezza) per identificare gli eventi associabili alle onde di Rayleigh. La prassi prevede che in questi casi, anziché i massimi di energia, vengano identificati sullo spettro i punti di transizione tra l'area ad energia maggiore ed il rumore incoerente di fondo. Questo serve a mitigare l'effetto dei segnali che si propagano non in linea con lo stendimento di misura e che producono una sovrastima delle velocità.

Per questo motivo tale tecnica risulta più soggettiva rispetto alle misure attive e alle misure passive con array bidimensionali. Inoltre fornisce risultati errati qualora il rumore provenga da una direzione preferenziale.

Per essere sicuri dell'attendibilità della prova occorrerebbe realizzare contestualmente un array bidimensionale e verificare la direzionalità del rumore. Questo non è quasi mai possibile (per ragioni di spazio) ed è molto dispendioso in termini di tempo. In quest'ottica è buona norma eseguire contestualmente una prova di tipo attivo (ad esempio di tipo MASW) sfruttando l'analogia tra gli schemi di acquisizione (si tratta sostanzialmente dello stesso stendimento). In questo modo è possibile verificare l'attendibilità di una prova RE.MI. almeno per la banda di frequenze in cui la curva stimata con una prova attiva si sovrappone a quella stimata con il RE.MI.

\subsubsection{Inversione}

La curva di dispersione sperimentale (eventualmente costituita da più modi e combinata con le curve stimate utilizzando prove superficiali attive), viene utilizzata per un processo di inversione che fornisce come risultato finale il profilo verticale di $\mathrm{V}_{\mathrm{s}}$. La risoluzione del problema inverso implica la parametrizzazione del modello di sottosuolo assunto, che viene di norma schematizzato come un mezzo elastico a strati piano-paralleli, omogenei e isotropi, nel quale l'eterogeneità è rappresentata dalla differenziazione delle caratteristiche meccaniche degli strati. I parametri di modello sono $\mathrm{V}_{\mathrm{S}}$, spessore e densità di ogni strato. I parametri incogniti dell'inversione sono $\mathrm{V}_{\mathrm{S}}$ ed eventualmente lo spessore degli strati, mentre e la densità sono assunti a priori. Inoltre, l'utilizzo di un modello monodimensionale è un'ipotesi che deve essere in linea di massima soddisfatta dal sito per garantire la validità del risultato finale.

Il processo di inversione è iterativo: a partire da un profilo di primo tentativo, costruito sulla base di metodi semplificati, ed eventualmente delle informazioni note a priori riguardo la stratigrafia, il problema diretto (simulazione della curva di dispersione teorica) viene risolto diverse volte variando i parametri incogniti di modello in modo da minimizzare lo scarto fra il set di dati sperimentali (curva di dispersione misurata) e il set di dati calcolati (curva di dispersione simulata). Il processo termina quando viene individuato un insieme di para- 
metri di modello che renda lo scarto accettabile. Il numero dei parametri di modello deve essere scelto come il minimo in grado di descrivere la curva di dispersione sperimentale (solitamente 2-3-4 strati). Una sovraparametrizzazione rischia, infatti, di ridurre la risoluzione dei singoli parametri dovuta a problemi di equivalenza. In particolare, qualora si invertano solo curve di dispersione ottenute da prove passive, quindi povere in contenuto di alte frequenze, è bene non sovraparametrizzare la parte più superficiale del profilo, in quanto la presenza di molti strati li renderebbe irrisolti.

La procedura di inversione può essere condotta con tecniche di ricerca locale (metodi linearizzati) o globale (metodi Monte Carlo) della soluzione.

\subsubsection{Documentazione}

La relazione in cui vengono riportate le metodologie di indagine, gli algoritmi impiegati, le analisi realizzate e i risultati ottenuti dovrà contenere:

- informazioni generali (commessa, cantiere, ubicazione possibilmente georeferenziata, data, nominativo dell'operatore, parametri di acquisizione e strumentazione utilizzata);

- $\quad$ sismogrammi originali su supporto magnetico;

- in caso di array di misura bidimensionali, immagini dello spettro tridimensionale (f-kxky) con evidenziati i massimi spettrali;

- $\quad$ in caso di prove RE.MI., immagini dello spettro bidimensionale ( $\mathrm{f}-\mathrm{k}, \mathrm{w}-\mathrm{p}, \mathrm{f}-\mathrm{v}$ ) con evidenziati i punti individuati per la stima della curva di dispersione e considerazioni sull'attendibilità della prova (confronto con dati attivi);

- confronto tra la curva stimata e quella sintetica generata a partire dal modello finale di $\mathrm{V}_{\mathrm{S}}$;

- confronto tra il profilo di $\mathrm{V}_{\mathrm{S}}$ di primo tentativo e quello finale del processo di inversione;

- tabelle con i parametri calcolati e parametri assunti a priori (spessore degli strati, $\mathrm{V}_{\mathrm{S}}$ e densità).

\subsubsection{Rilievo topografico del piano completo di prospezione sismica}

Come descritto precedentemente, per l'interpretazione delle misure di campagna si assume come modello del terreno un mezzo elastico-lineare a strati omogenei. Tale modello implica la necessità di avere un piano campagna sostanzialmente libero da ostacoli e privo di marcate irregolarità topografiche. Inoltre, per ottenere risultati significativi, è necessario che le condizioni geologiche del sottosuolo siano tali da soddisfare il modello di stratificazione sub-orizzontale.

\subsection{2. ildagini di sismica passiva (HVSR)}

\subsubsection{Generalità}

La metodologia, chiamata anche tecnica Nakamura (1989), è stata introdotta da Nogoshi e Igarashi (1971) sulla base di studi precedenti.
Questa tecnica si basa essenzialmente sul rapporto spettrale $\mathrm{H} / \mathrm{V}$ di rumore ambientale (seismic noise) e permette di valutare gli effetti locali di sito.

La tecnica proposta da Nakamura assume che i microtremori (il cosiddetto rumore di fondo registrabile in qualunque momento posizionando un sensore sismico sul terreno) consistano principalmente di un tipo di onde superficiali, le onde di Rayleigh, che si propagano in un singolo strato soffice su semispazio e che la presenza di questo strato sia la causa dell'amplificazione al sito.

\subsubsection{Caratteristiche delle attrezzature}

La misura prevede la registrazione, senza utilizzo di alcun strumento di energizzazione del terreno, del microtremore sismico ambientale nel dominio del tempo, sulle tre componenti dello spazio attraverso il posizionamento di adeguati strumenti sismometrici costituiti da sensori tridimensionali.

La strumentazione di acquisizione presenta le seguenti specifiche:

1. trasduttori tricomponenti (N-S, E-W, verticale) a bassa frequenza $(<1-2 \mathrm{~Hz})$

2. amplificatori;

3. digitalizzatore;

4. frequenza di campionamento: > $50 \mathrm{~Hz}$;

5. convertitore $\mathrm{A} / \mathrm{D}$ (analogico digitale) a $24 \mathrm{bit}$;

6. durata registrazione: $>15$ minuti;

7. collegamento al tempo GPS per la referenziazione temporale.

\subsubsection{Modalità esecutive}

Come accennato l'installazione lo strumento di misura dovrà essere orientato secondo le direzioni geografiche (E e W) e dovrà essere dotato di bolla sferica per il posizionamento mentre l'accoppiamento con la superficie dovrà essere diretto o assicurato con piedini o puntazze in terreni morbidi.

Bisognerà altresì fare attenzione alla presenza di radici, sottoservizi, vicinanza edifici, vento ecc., in quanto creano disturbo nel segnale $\mathrm{H} / \mathrm{V}$ inducendo una forte perturbazione a bassa frequenza.

Per uno studio di risposta di sito è consigliabile effettuare almeno tre misure ognuna di almeno 15-20 minuti per punto, possibilmente in tempi diversi durante la giornata, da cui derivare il valore di frequenza di risonanza.

\subsubsection{Elaborazione dei dati}

L'elaborazione dei dati raccolti deve impiegare un software in grado di consentire la determinazione delle frequenze di risonanza del sottosuolo mediante la tecnica dei rapporti spettrali secondo le linee guida del progetto europeo SESAME (Site EffectS assessment using Ambient Excitations, 2005).

Il processing dei dati verte sul rapporto spettrale tra il segnale del sensore verticale e quelli orizzontali operando su finestre di selezione del segnale che dovranno essere non meno di 10 per un segnale complessivo utile non inferiore a 200400 secondi. 
I principali passi del processing sono i seguenti:

1. FFT (incluso il tapering);

2. operatore di smoothing (Konno \& Ohmachi);

3. merging dei componenti orizzontali;

4. H/V Spectral Ratio per ogni finestra utilizzata $(>10)$;

5. media degli spettri $\mathrm{H} / \mathrm{V}$;

6. valutazione della deviazione standard.

Le risultanze dell'elaborazione sono presentate mediante graficazione dei rapporti spettrali $\mathrm{H} / \mathrm{V}$ delle varie componenti indicando il massimo del rapporto HVSR nel valore di $\mathrm{f}_{0}$ Frequenza/e di Risonanza e la sua deviazione standard.

\subsubsection{Documentazione}

Il certificato finale della prova presenta:

1. i criteri di attendibilità della misura;

2. i criteri di validità del picco di $\mathrm{f}_{0}$;

3. i valori di soglia delle condizioni di stabilità;

4. l'analisi dei criteri in particolare con verifica rispetto alla frequenza del sensore ed alla presenza di rumore di origine industriale;

5. l'interpretazione di $\mathrm{f}_{0}$ e dello spettro $\mathrm{H} / \mathrm{V}$ nei termini di caratteristiche del sito.

Per gli scopi e finalità dell'indagine le misure HVSR offrono la possibilità di determinare:

1. valutazione dell'omogeneità del sito rispetto alle frequenze di risonanza;

2. spessori della coltre di copertura. 